\title{
Dietary fat intake and ovarian cancer risk: a meta-analysis of epidemiological studies
}

\author{
Wenlong Qiu ${ }^{1}$, Heng Lu ${ }^{1}$, Yana $\mathbf{Q i}^{1}$ and Xiuwen Wang ${ }^{1}$ \\ ${ }^{1}$ Department of Oncology, Qilu Hospital, School of Medicine, Shandong University, Jinan, Shandong, China \\ Correspondence to: Xiuwen Wang, email: WangXiuwenQL@163.com \\ Keywords: meta-analysis, ovarian epithelial carcinoma, dietary fat \\ Received: November 25, 2015 Accepted: April 10,2016 Published: April 22, 2016
}

ABSTRACT

Observational studies assessing the association of dietary fat and risk of ovarian cancer yield discrepant results. Pertinent prospective cohort studies were identified by a PubMed search from inception to December 2015. Sixteen independent case-control and nine cohort studies on dietary fat intake were included, with approximately 900,000 subjects in total. Relative risks (RRs) with $95 \%$ confidence intervals were pooled using a random effects model. Heterogeneity, sensitivity analysis and publication bias were assessed; subgroup analysis and analysis stratified by EOC histology were conducted. The reported studies showed a significant increase of ovarian cancer risk with high consumption of total-, saturated-, and trans-fats, while serous ovarian cancer was more susceptible to dietary fat consumption than other pathological subtypes. No evidence of positive association between dietary fat intake and ovarian cancer risk was provided by cohort studies. Menopausal status, hormone replacement therapy, body mass index (BMI), and pregnancy times, modified the objective associations. In conclusion, the meta-analysis findings indicate that high consumption of total, saturated and trans-fats increase ovarian cancer risk, and different histological subtypes have different susceptibility to dietary fat.

\section{INTRODUCTION}

Ovarian cancer is considered the sixth most commonly diagnosed cancer among women and the second cause of gynecologic cancer mortality worldwide $[1,2]$. The prognosis of ovarian cancer is poor, with the initial diagnosis in most patients made at an advanced stage $[3,4]$. The noticeable relationship between ovarian cancer incidence and geographical regions suggested that dietary habits and ethnic variations are potentially modifiable factors [5], whose etiologic role in ovarian cancer risk, however, remains undefined [6].

Dietary fat, as one of the most controversial dietary factors in nutritional epidemiology, has been reported with positive correlations with breast [7] and gastric [8] cancers in two recent meta-analyses, and elevated ovarian cancer risk in early ecologic studies [5,9]. Although multiple epidemiologic studies have explored the associations between dietary fat consumption and risk of ovarian cancer, no definite conclusion have been drawn, and the dietary fat varieties as well as pathological types of ovarian cancer increase the complexity of this research topic. The results of two meta-analyses $[10,11]$ and a pooled analysis [12] that included data from 12 cohort studies also reached inconsistent conclusions. Therefore, we conducted a meta-analysis of case-control and cohort studies with more-detailed analyses of 1) the epidemiologic evidence regarding the association of dietary fat consumption with risk of ovarian cancer, 2) the association between dietary fat intake and the risk of ovarian cancer and pathological subtypes. This analysis was based on dietary fat types, and we extended the previous analyses $[10,11]$ with more included studies and dietary fat types, and an assessment stratified by EOC histology.

\section{RESULTS}

We found 1421 publications from the electronic and manual literature searches. Thirty-three potentially relevant publications [16-31] [32-47] [48] appeared to 
meet the specified protocol inclusion criteria after initial screening. Through further reading, six publications [4146] contained no relevant dietary fat; two publications [47, 48] were excluded for design and dietary fat classification. Two American publications [17, 18] assessed the same study population, and eligible data were extracted from both; two Chinese publications were treated likewise [25, 28] (Figure 1).

Finally, Twenty-five publications [16-31] [32-40], including sixteen case-control [16-31] (Table 1) and nine cohort [32-40] (Table 2) studies were included in the analysis of dietary fat intake and ovarian cancer risk. Results were separated by dietary fat types, including total, saturated, monounsaturated, polyunsaturated, animal, plant, dairy fat, and trans-fats.

\section{Total fat}

Eleven case-control and six cohort studies assessed total fat intake and ovarian cancer risk. Summary RR was $1.32(95 \% \mathrm{CI}=1.06-1.63, P=0.017)$ for case-control and $1.10(95 \% \mathrm{CI}=0.97-1.24, P=0.25)$ for cohort studies, with an overall RR of $1.19(95 \% \mathrm{CI}=1.04-1.37, P=$ 0.015 ) for all studies. These results suggested a positive association between total fat intake and ovarian cancer risk. (Figure 2)

\section{Animal fat}

Five case-control and five cohort studies analyzed animal fat intake and ovarian cancer risk. Summary RR was $1.50(95 \% \mathrm{CI}=0.89-2.53, P=0.125)$ for case-control, and $1.09(95 \% \mathrm{CI}=0.93-1.28, P=0.272)$ for cohort studies; the overall RR was $1.21(95 \% \mathrm{CI}=0.99-1.47$, $P=0.065)$ for all studies. Taken together, these results suggested no association between animal fat intake and ovarian cancer risk. (Figure 3)

\section{Plant fat}

Four case-control and five cohort studies evaluated plant fat intake and ovarian cancer risk. Summary RR was $0.96(95 \% \mathrm{CI}=0.81-1.12, P=0.586)$ for case-control, and $0.93(95 \% \mathrm{CI}=0.74-1.17, P=0.053)$ for cohort studies, with an overall RR of $0.95(95 \% \mathrm{CI}=0.83-1.09, P=$ 0.472 ). These results suggested no association between plant fat intake and ovarian cancer risk. (Figure 4)

\section{Saturated fat}

Seven case-control and six cohort studies assessed saturated fat intake and ovarian risk. Summary RR was $1.11(95 \% \mathrm{CI}=0.98-1.27, P=0.147)$ for case-control, and $1.06(95 \% \mathrm{CI}=0.89-1.26, P=0.0 .521)$ for cohort studies; the overall RR was 1.09 (95\% CI $=0.98-1.21$, $P=0.103$ ). After exclusion of 1 study [29] for small bias and sensitivity data, the results changed as follows, respectively, $1.15(95 \% \mathrm{CI}=1.02-1.30, P=0.026), 1.06$ (95\% CI $=0.89-1.26, P=0.521), 1.12$ (95\%CI $=1.02$ $1.22, P=0.014)$, without heterogeneity $(\mathrm{Q}=8.54, P=$ $0.577, \mathrm{I} 2=0.0 \%$ ) between studies. These results suggested a positive association between saturated fat intake and ovarian cancer risk. (Figure 5)

\section{Monounsaturated fat}

Eight case-control and five cohort studies analyzed monounsaturated fat intake and ovarian cancer risk. Summary RR was $0.96(95 \% \mathrm{CI}=0.83-1.12, P=0.477)$ for case-control, and $1.04(95 \% \mathrm{CI}=0.88-1.22, P=$ $0.649)$ for cohort studies; the overall RR was $0.98(95 \%$ $\mathrm{CI}=0.87-1.09, P=0.556)$ for all studies. These results suggested no association between total fat intake and ovarian cancer risk. (Figure 6)

\section{Polyunsaturated fat}

Eight case-control and five cohort studies involved polyunsaturated fat intake and ovarian cancer risk. Summary RR was $0.92(95 \% \mathrm{CI}=0.81-1.04, P=0.223)$ for case-control, and $1.06(95 \% \mathrm{CI}=0.86-1.31, P=0.570)$ for cohort studies, with an overall RR of 0.97 (95\% CI $=0.86-1.10, P=0.760$ ) for all studies. These findings suggested no association between polyunsaturated fat intake and ovarian cancer risk. (Figure 7)

\section{Dairy fat}

One case-control and five cohort studies assessed dairy fat intake and the risk of ovarian cancer. Summary RR was $1.10(95 \% \mathrm{CI}=0.94-1.28, P=0.242)$ for cohort studies, with an overall RR of $1.05(95 \% \mathrm{CI}=0.92-1.19$, $P=0.478)$ for all studies. These results suggested no association between dairy fat intake and ovarian cancer risk. (Figure 8)

\section{Trans-fat}

Two case-control and two cohort studies evaluated trans-fat intake and ovarian cancer risk. Summary RR was $1.25(95 \% \mathrm{CI}=1.06-1.49, P=0.010)$ for case-control, and $1.24(95 \% \mathrm{CI}=0.85-1.81, P=0.285)$ for cohort studies; the overall RR was $1.25(95 \% \mathrm{CI}=1.08-1.44, P=0.002)$ for all studies. These results suggested a significant positive association between trans-fat intake and ovarian cancer risk. (Figure 9) 
Table 1:The characteristics and relative risks [RRs; $95 \%$ confidence interval (CI)] for case-control studies on dietary fat and ovarian cancer

\begin{tabular}{|c|c|c|c|c|c|}
\hline $\begin{array}{l}\text { Author } \\
\text { (Country) }\end{array}$ & $\begin{array}{l}\text { Publication } \\
\text { yr }\end{array}$ & Case/Control & Type of Fat & RR (95\% CI) & $\begin{array}{l}\begin{array}{l}\text { Adjusted confounding } \\
\text { factors }\end{array} \\
\end{array}$ \\
\hline $\begin{array}{l}\text { Cramer[16] } \\
\text { (America) }\end{array}$ & $\begin{array}{l}1984 \\
(1978-1981)\end{array}$ & $215 / 215$ & Animal fat & $1.83(1.00-3.38)$ & Weight/height ${ }^{2}$ \\
\hline $\begin{array}{l}\text { Risch }[17,18] \\
\text { (Canada) }\end{array}$ & $\begin{array}{l}1994 \\
(1989-1992)\end{array}$ & $450 / 564$ & $\begin{array}{l}\text { Total fat } \\
\text { Saturated fat } \\
\text { Monounsaturated fat } \\
\text { Polyunsaturated fat } \\
\end{array}$ & \begin{tabular}{|l}
$1.16(0.86-1.57)$ \\
$1.23(0.97-1.58)$ \\
$1.07(0.90-1.27)$ \\
$0.86(0.69-1.07)$ \\
\end{tabular} & $\begin{array}{l}\text { Age, total calorie intake, } \\
\text { no. of } \\
\text { full-term pregnancies, } \\
\text { duration of OC use }\end{array}$ \\
\hline $\begin{array}{l}\text { Shu[19] } \\
\text { (China) }\end{array}$ & $\begin{array}{l}1989 \\
(1984-1986)\end{array}$ & $172 / 172$ & $\begin{array}{l}\text { Total fat } \\
\text { Animal fat } \\
\text { Plant fat }\end{array}$ & $\begin{array}{l}1.9(1.2-4.4) \\
1.7(1.0-3.2) \\
0.8(0.4-1.4)\end{array}$ & $\begin{array}{l}\text { income, no. of live } \\
\text { births, history of ovarian } \\
\text { cysts, smoking history, } \\
\text { OC use, IUD use, tubal } \\
\text { ligation }\end{array}$ \\
\hline $\begin{array}{l}\text { Slattery[20] } \\
\text { (America) }\end{array}$ & $\begin{array}{l}1989 \\
(1984-1987)\end{array}$ & $85 / 492$ & $\begin{array}{l}\text { Total fat } \\
\text { Saturated fat } \\
\text { Monounsaturated fat } \\
\text { Polyunsaturated fat } \\
\end{array}$ & $\begin{array}{l}1.3(0.7-2.3) \\
1.3(0.6-2.6) \\
1.3(0.7-2.3) \\
1.2(0.6-2.3) \\
\end{array}$ & $\begin{array}{l}\text { Age, BMI, no. of } \\
\text { pregnancies }\end{array}$ \\
\hline $\begin{array}{l}\text { Tzonou[21] } \\
\text { (Greece) }\end{array}$ & $\begin{array}{l}1993 \\
(1989-1991)\end{array}$ & $189 / 200$ & $\begin{array}{l}\text { Total fat } \\
\text { Saturated fat } \\
\text { Monounsaturated fat } \\
\text { Polyunsaturated fat } \\
\end{array}$ & \begin{tabular}{|l|}
$0.97(0.76-1.24)$ \\
$1.17(0.88-1.55)$ \\
$0.86(0.71-1.05)$ \\
$0.95(0.77-1.17)$ \\
\end{tabular} & $\begin{array}{l}\text { Age, years of schooling, } \\
\text { parity, age at 1st birth, } \\
\text { menopausal status, } \\
\text { energy intake }\end{array}$ \\
\hline $\begin{array}{l}\text { La Vecchia[22] } \\
\text { (Italy) }\end{array}$ & $\begin{array}{l}1987 \\
(1983-1986)\end{array}$ & $455 / 1385$ & Total fat & $2.14(1.59-2.88)$ & $\begin{array}{l}\text { Age, interviewer, } \\
\text { marital status, social } \\
\text { class, education, parity, } \\
\text { age at 1st birth, age at } \\
\text { menarche, menopausal } \\
\end{array}$ \\
\hline $\begin{array}{l}\text { Webb[23] } \\
\text { (Australia) }\end{array}$ & $\begin{array}{l}1998 \\
(1990-1993)\end{array}$ & $824 / 1132$ & Total fat & $1.86(1.03-3.37)$ & $\begin{array}{l}\text { Age, education, BMI, } \\
\text { smoking, parity, OC use, } \\
\text { total energy intake }\end{array}$ \\
\hline $\begin{array}{l}\text { Pan }[24] \\
\text { (Canada) }\end{array}$ & $\begin{array}{l}2004 \\
(1994-1997)\end{array}$ & $442 / 2135$ & $\begin{array}{l}\text { Total fat } \\
\text { Saturated fat } \\
\text { Monounsaturated fat } \\
\text { Polyunsaturated fat }\end{array}$ & $\begin{array}{l}1.21(0.88-1.65) \\
1.06(0.78-1.45) \\
1.26(0.92-1.72) \\
1.28(0.94-1.76)\end{array}$ & $\begin{array}{l}\text { Age, residence, } \\
\text { education, alcohol } \\
\text { consumption, smoking, } \\
\text { BMI, caloric intake, } \\
\text { recreational physical } \\
\text { activity, number of live } \\
\text { births, menstruation } \\
\text { years, and menopause } \\
\text { status }\end{array}$ \\
\hline $\begin{array}{l}\text { Zhang[25] } \\
\text { (China) }\end{array}$ & $\begin{array}{l}2002 \\
(1999-2000)\end{array}$ & $254 / 652$ & $\begin{array}{l}\text { Animal fat } \\
\text { Plant fat }\end{array}$ & $\begin{array}{l}4.55(2.2-9.3) \\
1.03(0.6-1.9)\end{array}$ & $\begin{array}{l}\text { Age, residence, } \\
\text { education, alcohol } \\
\text { consumption, smoking, } \\
\text { BMI, tube ligation, } \\
\text { menopause status }\end{array}$ \\
\hline $\begin{array}{l}\text { McCann[26] } \\
\text { (America) }\end{array}$ & $\begin{array}{l}2003 \\
(1986-1991)\end{array}$ & $124 / 696$ & $\begin{array}{l}\text { Total fat } \\
\text { Saturated fatty acid } \\
\text { Monounsaturated fat } \\
\text { Polyunsaturated fat }\end{array}$ & $\begin{array}{l}1.51(0.57-4.02) \\
1.46(0.68-3.15) \\
1.77(0.73-4.31) \\
0.63(0.28-1.41)\end{array}$ & $\begin{array}{l}\text { Age, education, total } \\
\text { months menstruating, } \\
\text { difficulty becoming } \\
\text { pregnant, OC use, } \\
\text { menopausal status and } \\
\text { total energy intake }\end{array}$ \\
\hline $\begin{array}{l}\text { Merritt[27] } \\
\text { (America) }\end{array}$ & $\begin{array}{l}2014 \\
(1992-2008)\end{array}$ & $1872 / 1978$ & $\begin{array}{l}\text { Total fat } \\
\text { Animal fat } \\
\text { Dairy fat } \\
\text { Saturated fat } \\
\text { Plant fat } \\
\text { Trans fat } \\
\text { Monounsaturated fat } \\
\text { Polyunsaturated fat } \\
\end{array}$ & \begin{tabular}{|l}
$1.07(0.89-1.29)$ \\
$1.04(0.87-1.26)$ \\
$0.95(0.79-1.14)$ \\
$1.11(0.92-1.34)$ \\
$0.98(0.81-1.17)$ \\
$1.30(1.08-1.57)$ \\
$0.97(0.81-1.18)$ \\
$0.82(0.68-0.99)$ \\
\end{tabular} & $\begin{array}{l}\text { Age, study centre (MA, } \\
\text { NH), study phase, } \\
\text { number of pregnancies, } \\
\text { OC use, family history } \\
\text { of ovarian cancer tubal } \\
\text { ligation }\end{array}$ \\
\hline
\end{tabular}




\begin{tabular}{|c|c|c|c|c|c|}
\hline $\begin{array}{l}\text { Zhang }[28] \\
\text { (China) }\end{array}$ & $\begin{array}{l}2003 \\
(1999-2000)\end{array}$ & $254 / 652$ & Total fat & $2.17(1.26-3.75)$ & $\begin{array}{l}\text { Age, locality, education, } \\
\text { family income, BMI, } \\
\text { total energy intake, } \\
\text { tobacco smoking, alcohol } \\
\text {,parity, menopausal } \\
\text { status, OC use }\end{array}$ \\
\hline $\begin{array}{l}\text { Salazar- } \\
\text { Martinez[29] } \\
\text { (Mexico) }\end{array}$ & $\begin{array}{l}2002 \\
(1995-1997)\end{array}$ & $84 / 629$ & \begin{tabular}{|l} 
Total fat \\
Saturated fat \\
Monounsaturated fat \\
Polyunsaturated fat \\
Animal fat \\
Plant fat \\
\end{tabular} & \begin{tabular}{|l}
$0.60(0.33-1.06)$ \\
$0.56(0.31-1.02)$ \\
$0.54(0.30-0.99)$ \\
$0.61(0.34-1.11)$ \\
$0.66(0.37-1.19)$ \\
$0.81(0.46-1.45)$ \\
\end{tabular} & $\begin{array}{l}\text { Age, weight change, total } \\
\text { energy intake, number } \\
\text { of live births, physical } \\
\text { activity, diabetes }\end{array}$ \\
\hline $\begin{array}{l}\text { Chiaffarino[30] } \\
\text { (Italy) }\end{array}$ & $\begin{array}{l}2007 \\
(1992-1999)\end{array}$ & $750 / 2411$ & $\begin{array}{l}\text { Monounsaturated fat } \\
\text { Polyunsaturated fat }\end{array}$ & $\begin{array}{l}0.80(0.66-0.96) \\
0.96(0.76-1.21)\end{array}$ & $\begin{array}{l}\text { education, parity, oral } \\
\text { contraceptive use, family } \\
\text { history of ovarian and/ } \\
\text { or breast cancer in first } \\
\text { degree relatives }\end{array}$ \\
\hline $\begin{array}{l}\mathrm{Hu}[31] \\
\text { (Canada) }\end{array}$ & $\begin{array}{l}2011 \\
(1994-1997)\end{array}$ & $442 / 5039$ & Trans fat & $1.04(0.68-1.58)$ & $\begin{array}{l}\text { Age, province, } \\
\text { education, BMI, alcohol } \\
\text { drinking, } \\
\text { pack-year smoking, total } \\
\text { of vegetable } \\
\text { and fruit intake, } \\
\text { monounsaturated fat, } \\
\text { polyunsaturated fat, total } \\
\text { energy intake, number of } \\
\text { live births and years of } \\
\text { menstruation }\end{array}$ \\
\hline
\end{tabular}

Potentially relevant studies identified through PubMed search $(\mathrm{n}=1421)$

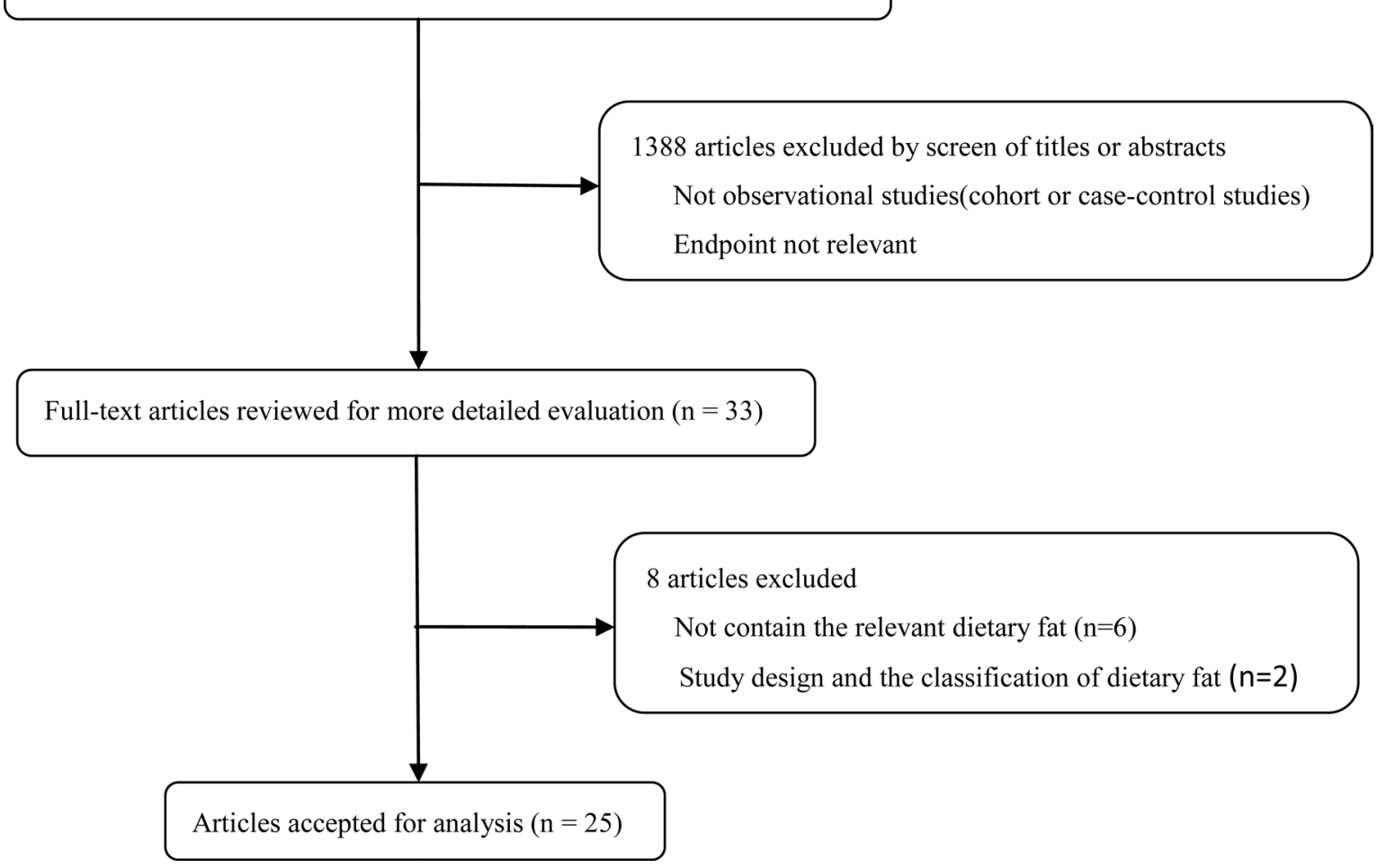

Figure 1: Flow chart. 


\section{Total Fat and Ovarian Cancer Risk}

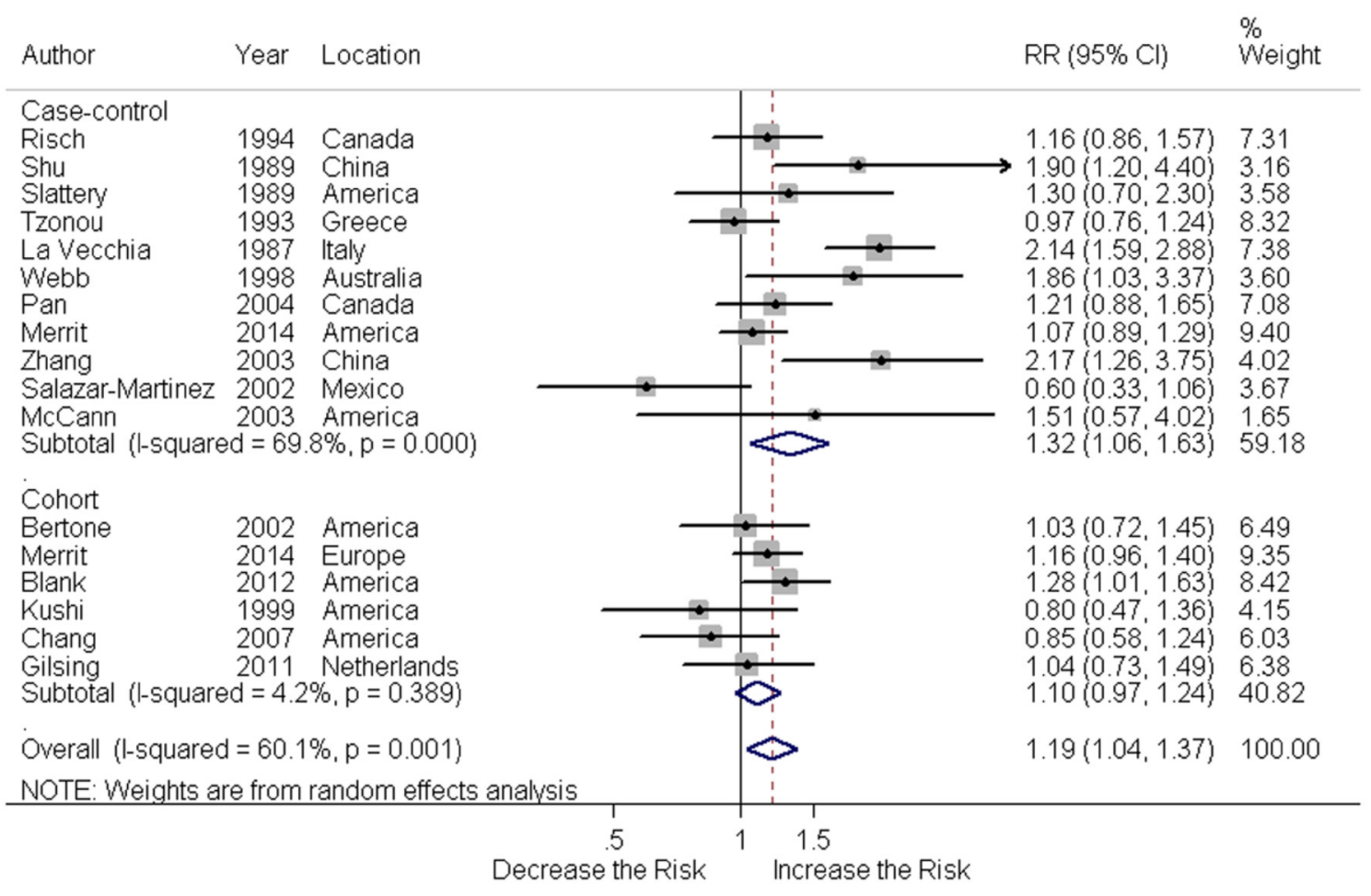

Figure 2: Relationship between total fat intake and ovarian cancer risk.

\section{Animal Fat and Ovarian Cancer Risk}

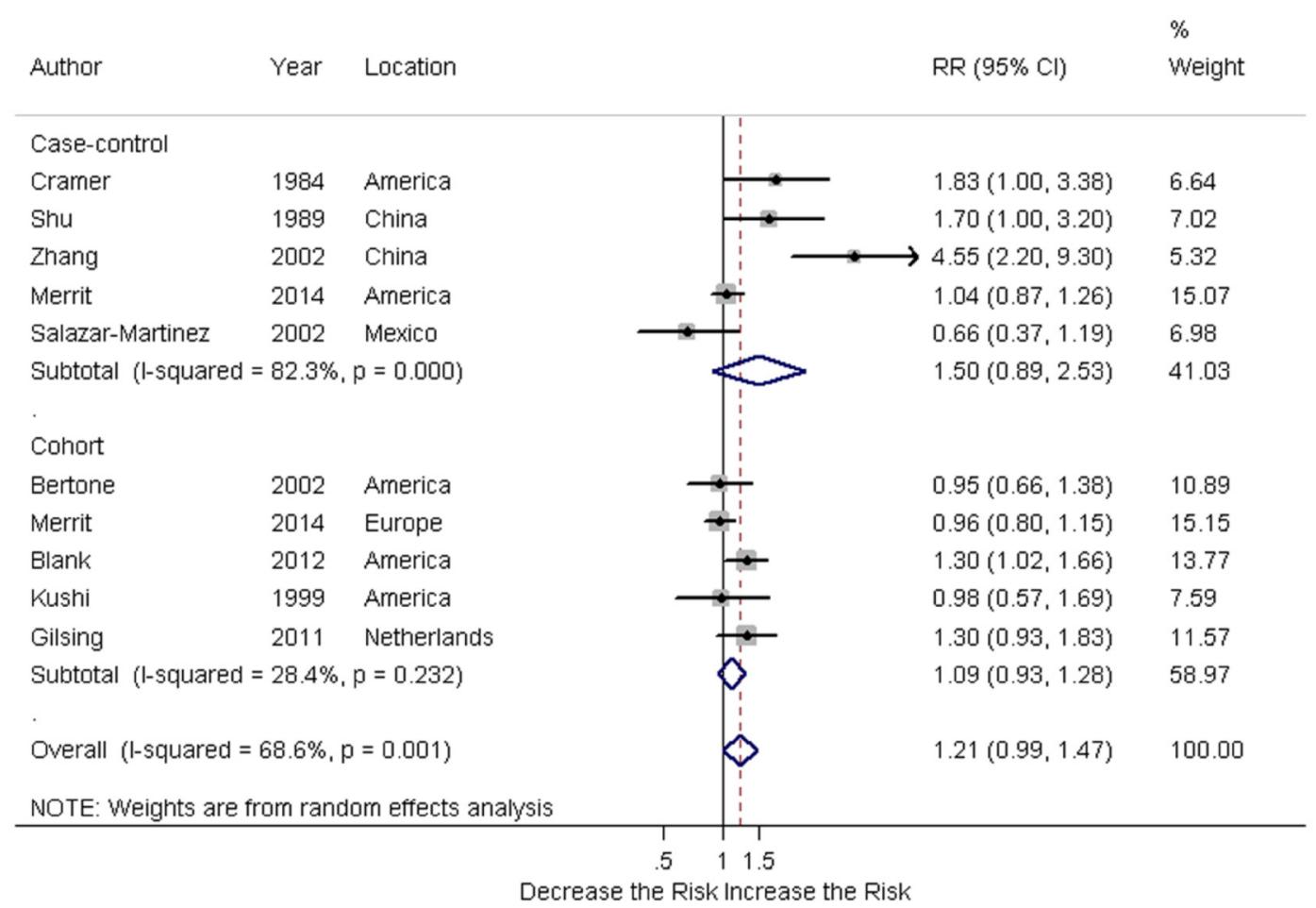

Figure 3: Relationship between animal fat intake and ovarian cancer risk. 


\section{Plant Fat and Ovarian Cancer Risk}

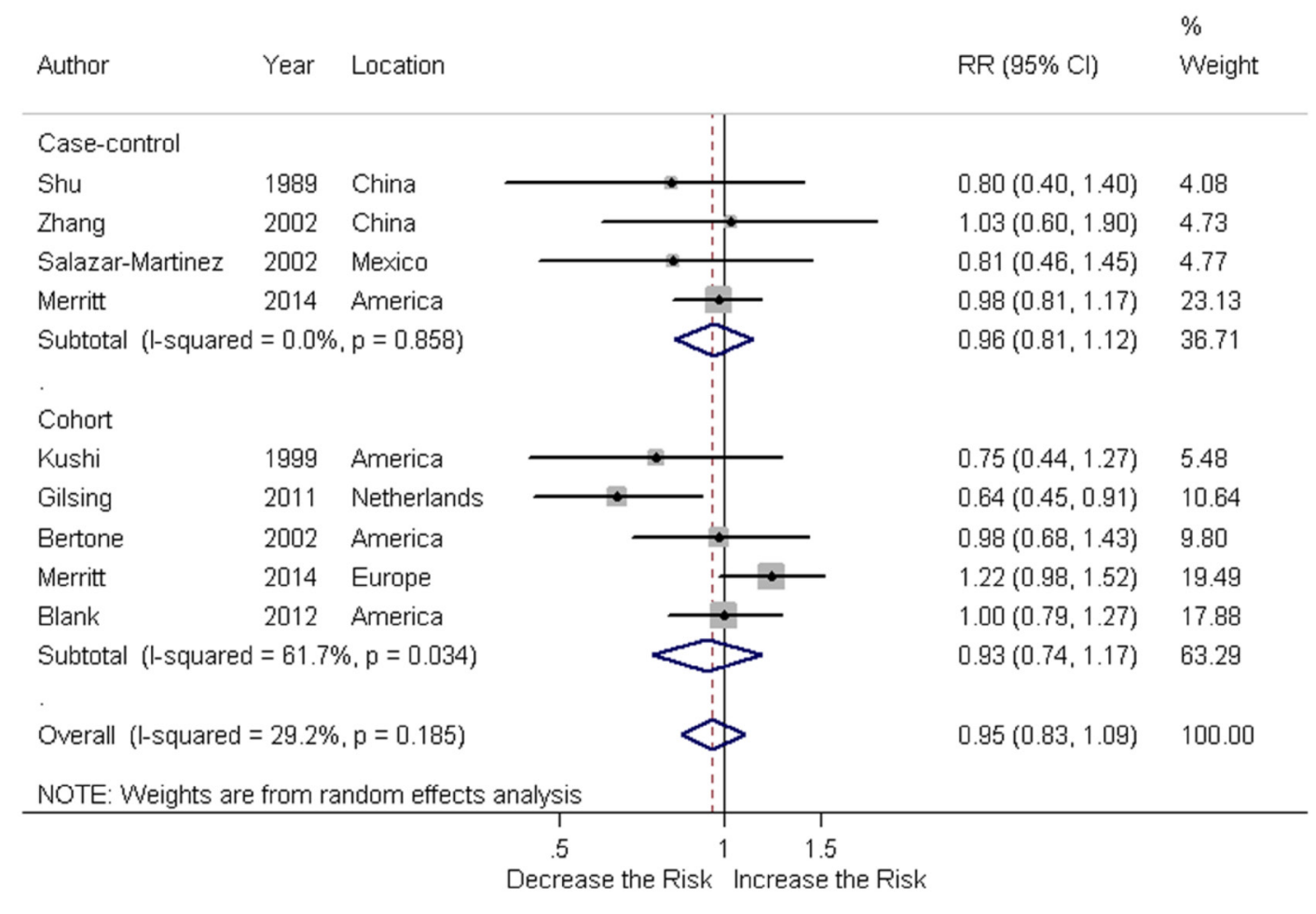

Figure 4: Relationship between plant fat intake and ovarian cancer risk.

\section{Saturated Fat and Ovarian Cancer Risk}

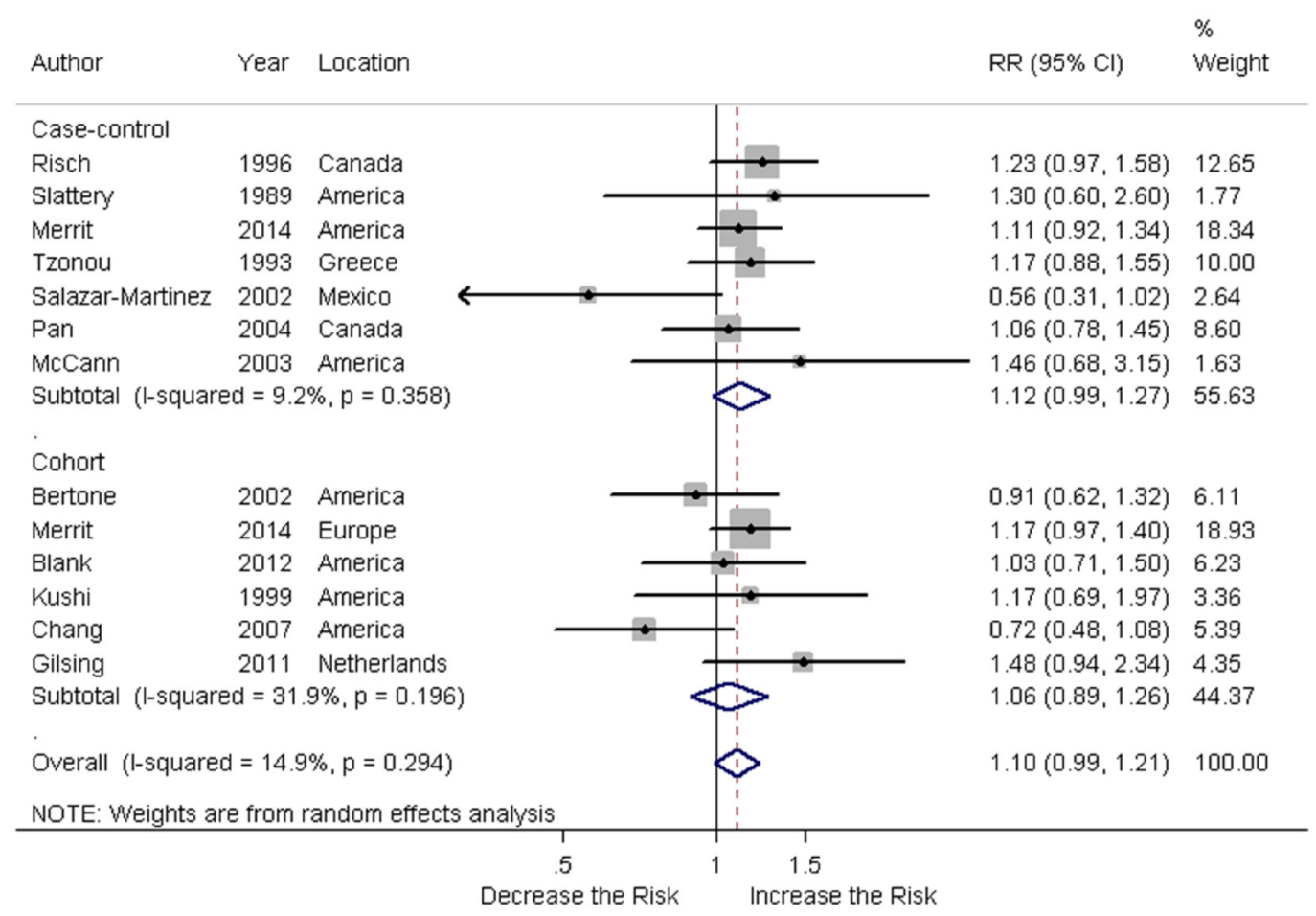

Figure 5: Relationship between saturated fat intake and ovarian cancer risk. 


\section{Monounsaturated Fat and Ovarian Cancer Risk}

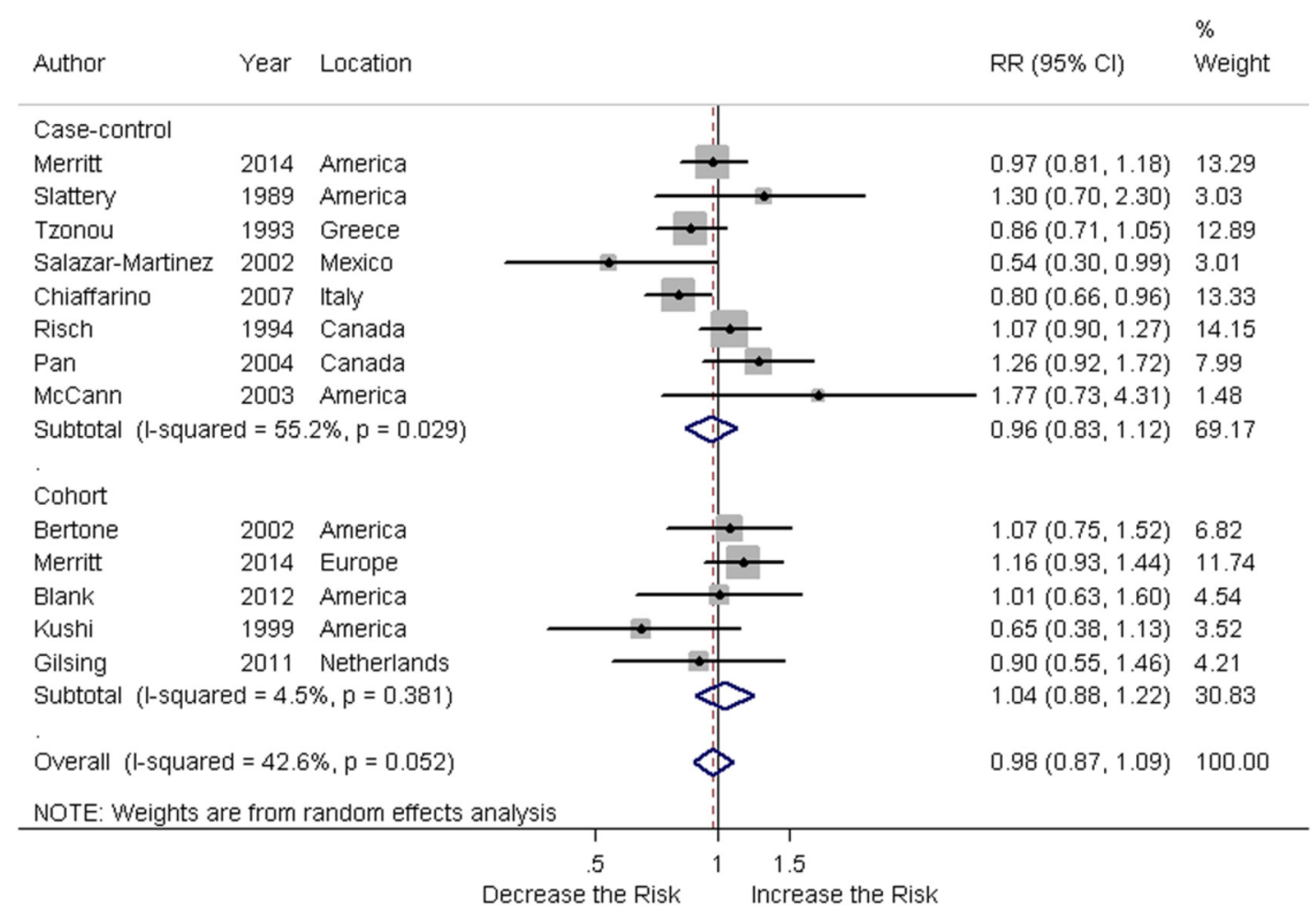

Figure 6: Relationship between monounsaturated fat intake and ovarian cancer risk.

\section{Polyunsaturated Fat and Ovarian Cancer Risk}

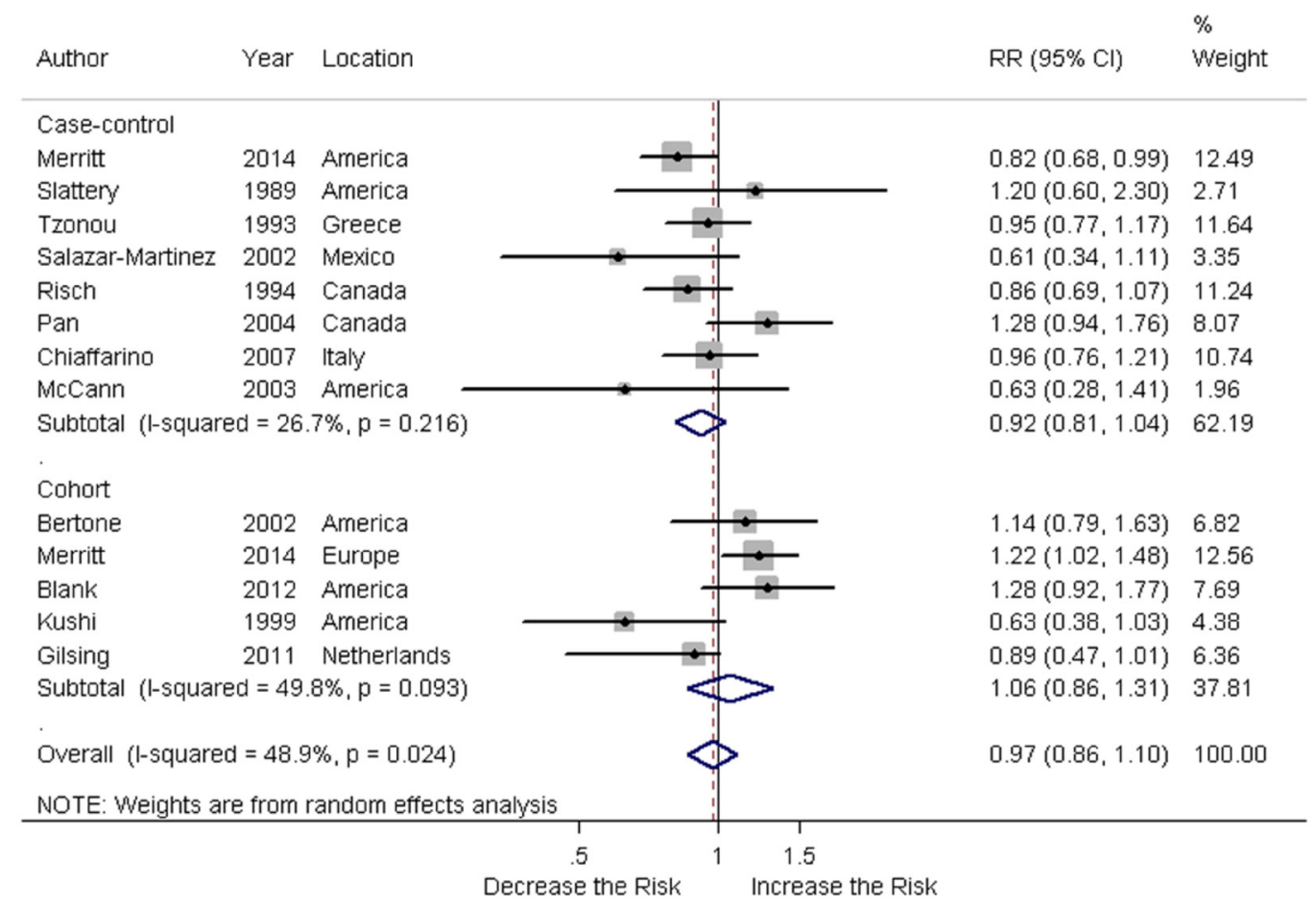

Figure 7: Relationship between polyunsaturated fat intake and ovarian cancer risk. 
Table 2: The characteristics and relative risks [RRs; $95 \%$ confidence interval (CI)] for cohort studies on dietary fat and ovarian cancer

\begin{tabular}{|c|c|c|c|c|c|}
\hline \begin{tabular}{|l|} 
Author \\
(Country)
\end{tabular} & Publication yr & Case/Total & Type of Fat & RR $(95 \%$ CI $)$ & \begin{tabular}{|ll}
$\begin{array}{l}\text { Adjusted } \\
\text { factors }\end{array}$ & confounding \\
\end{tabular} \\
\hline $\begin{array}{l}\text { Merritt[32] } \\
\text { (America) }\end{array}$ & $\begin{array}{l}2014 \\
(1980-2009)\end{array}$ & 764/95452 & Dairy fat & $1.01(0.80-1.27)$ & $\begin{array}{l}\text { Caloric intake, number of } \\
\text { pregnancies, parity, OC } \\
\text { use, menopausal status, } \\
\text { tubal ligation ,family } \\
\text { history }\end{array}$ \\
\hline $\begin{array}{l}\text { Bertone[33] } \\
\text { (America) }\end{array}$ & $\begin{array}{l}2002 \\
(1980-1996)\end{array}$ & $301 / 80258$ & $\begin{array}{l}\text { Total fat } \\
\text { Animal fat } \\
\text { Dairy fat } \\
\text { Saturated fat } \\
\text { Plant fat } \\
\text { Monounsaturated fat } \\
\text { Polyunsaturated fat } \\
\text { Trans fat } \\
\end{array}$ & $\begin{array}{l}1.03(0.72-1.45) \\
0.95(0.66-1.38) \\
1.06(0.73-1.54) \\
0.91(0.62-1.32) \\
0.98(0.68-1.43) \\
1.07(0.75-1.52) \\
1.14(0.79-1.63) \\
1.03(0.72-1.47)\end{array}$ & $\begin{array}{l}\text { Age, parity, age at } \\
\text { menarche, OC use, } \\
\text { menopausal ratu, } \\
\text { postmenopausal hormone } \\
\text { use, tubal ligation, } \\
\text { smoking status }\end{array}$ \\
\hline $\begin{array}{l}\text { Merritt[34] } \\
\text { (Europe) }\end{array}$ & 2014 & $1095 / 325007$ & $\begin{array}{l}\text { Total fat } \\
\text { Animal fat } \\
\text { Plant fat } \\
\text { Saturated fat } \\
\text { Monounsaturated fat } \\
\text { Polyunsaturated fat }\end{array}$ & $\begin{array}{l}1.16(0.96-1.40) \\
0.96(0.80-1.15) \\
1.22(0.98-1.52) \\
1.17(0.97-1.40) \\
1.16(0.93-1.44) \\
1.22(1.02-1.48)\end{array}$ & $\begin{array}{l}\text { OC use, number of } \\
\text { children, menopausal } \\
\text { status at enrolment, total } \\
\text { energy intake }\end{array}$ \\
\hline $\begin{array}{l}\text { Blank[35] } \\
\text { (America) }\end{array}$ & $\begin{array}{l}2012 \\
(1995-2005)\end{array}$ & $695 / 151552$ & $\begin{array}{l}\text { Total fat } \\
\text { Animal fat } \\
\text { Plant fat } \\
\text { Saturated fat } \\
\text { Monounsaturated fat } \\
\text { Polyunsaturated fat } \\
\end{array}$ & $\begin{array}{l}1.28(1.01-1.63) \\
1.30(1.02-1.66) \\
1.00(0.79-1.27) \\
1.03(0.71-1.50) \\
1.01(0.63-1.60) \\
1.28(0.92-1.77)\end{array}$ & $\begin{array}{l}\text { Age, race, education, } \\
\text { BMI, family history, OC } \\
\text { use, parity, menopausal } \\
\text { hormone therapy use, total } \\
\text { energy intake }\end{array}$ \\
\hline $\begin{array}{l}\text { Kiani[36] } \\
\text { (America) }\end{array}$ & $\begin{array}{l}2006 \\
(1976-1992)\end{array}$ & $71 / 13,281$ & Dairy fat & $0.94(0.70-1.27)$ & $\begin{array}{l}\text { Age, parity and BMI, and } \\
\text { also for age at menopause } \\
\text { and hormone replacement } \\
\text { therapy in postmenopausal } \\
\text { analyses }\end{array}$ \\
\hline $\begin{array}{l}\text { Mommers[37] } \\
\text { (Netherlands) }\end{array}$ & $\begin{array}{l}2006 \\
(1986-1997)\end{array}$ & $252 / 2216$ & Dairy fat & $1.53(1.00-2.36)$ & $\begin{array}{l}\text { Age, height, smoking, } \\
\text { number of cigarettes } \\
\text { smoked daily, OC use and } \\
\text { parity, and dairy products }\end{array}$ \\
\hline $\begin{array}{l}\text { Kushi[38] } \\
\text { (America) }\end{array}$ & $\begin{array}{l}1999 \\
(1986-1995)\end{array}$ & $139 / 29,083$ & $\begin{array}{l}\text { Total fat } \\
\text { Animal fat } \\
\text { Saturated fat } \\
\text { Plant fat } \\
\text { Monounsaturated fat } \\
\text { Polyunsaturated fat }\end{array}$ & $\begin{array}{l}0.80(0.47-1.36) \\
0.98(0.57-1.69) \\
1.17(0.69-1.97) \\
0.75(0.44-1.27) \\
0.65(0.38-1.13) \\
0.63(0.38-1.03)\end{array}$ & $\begin{array}{l}\text { Age, total energy intake, } \\
\text { no. of live births, age } \\
\text { at menopause, family } \\
\text { history of ovarian cancer, } \\
\text { hysterectomy, waist- } \\
\text { to-hip ratio, level of } \\
\text { physical activity, smoking, } \\
\text { education }\end{array}$ \\
\hline $\begin{array}{l}\text { Chang[39] } \\
\text { (America) }\end{array}$ & $\begin{array}{l}2007 \\
(1995-2003)\end{array}$ & $280 / 97275$ & $\begin{array}{l}\text { Total fat } \\
\text { Saturated fat }\end{array}$ & $\begin{array}{l}0.85(0.58-1.24) \\
0.72(0.48-1.08)\end{array}$ & $\begin{array}{l}\text { Race, energy intake, parity, } \\
\text { OC use, exercise, wine } \\
\text { consumption, menopausal } \\
\text { status, hormone therapy }\end{array}$ \\
\hline $\begin{array}{l}\text { Gilsing[40] } \\
\text { (Netherlands) }\end{array}$ & $\begin{array}{l}2011 \\
(1986-2002)\end{array}$ & $340 / 62,573$ & $\begin{array}{l}\text { Total fat } \\
\text { Animal fat } \\
\text { Plant fat } \\
\text { Dairy fat } \\
\text { Saturated fat } \\
\text { Monounsaturated fat } \\
\text { Polyunsaturated fat } \\
\text { Trans fat }\end{array}$ & $\begin{array}{l}1.04(0.73-1.49) \\
1.30(0.93-1.83) \\
0.64(0.45-0.91) \\
1.28(0.91-1.80) \\
1.48(0.94-2.34) \\
0.90(0.55-1.46) \\
0.89(0.47-1.01) \\
1.51(1.04-2.20)\end{array}$ & $\begin{array}{l}\text { Age, total energy intake, } \\
\text { parity (number of } \\
\text { children), and use of oral } \\
\text { contraceptives use }\end{array}$ \\
\hline
\end{tabular}


Table 3: Relative risks (RR) of ovarian cancer and corresponding $95 \%$ confidence intervals (CI) according to histological types and intake of dietary fat

\begin{tabular}{|c|c|c|c|c|c|c|c|c|}
\hline \multirow{3}{*}{$\begin{array}{l}\text { Types of dietary } \\
\text { fat }\end{array}$} & \multicolumn{8}{|c|}{ Histological types } \\
\hline & \multicolumn{2}{|l|}{ Serous tumor } & \multicolumn{2}{|c|}{ Endometroid tumor } & \multicolumn{2}{|c|}{ Mucinous tumor } & \multicolumn{2}{|l|}{ Other tumor } \\
\hline & RR $(95 \%$ CI) & $P$ & RR $(95 \% C I)$ & $P$ & RR $(95 \% C I)$ & $P$ & RR $(95 \% C I)$ & $P$ \\
\hline Total fat & $1.12(1.01,1.24)$ & 0.025 & $1.05(0.85,1.30)$ & 0.628 & $1.15(0.69,1.90)$ & 0.591 & $1.17(0.69,1.99)$ & 0.561 \\
\hline Animal fat & $1.07(1.00,1.16)$ & 0.061 & $0.96(0.79,1.17)$ & 0.715 & $1.36(1.08,1.73)$ & 0.011 & $0.82(0.49,1.38)$ & 0.456 \\
\hline Plant fat & $1.07(0.95,1.21)$ & 0.278 & $0.92(0.76,1.11)$ & 0.391 & $0.96(0.73,1.27)$ & 0.778 & $1.22(0.71,2.09)$ & 0.468 \\
\hline Saturated fat & $1.26(1.05,1.52)$ & 0.012 & $1.34(1.08,1.66)$ & 0.008 & $1.29(0.93,1.81)$ & 0.132 & $1.19(0.40,3.51)$ & 0.754 \\
\hline $\begin{array}{l}\text { Monounsaturated } \\
\text { fat }\end{array}$ & $0.97(0.82,1.14)$ & 0.692 & $0.78(0.57,1.06)$ & 0.115 & $1.09(0.88,1.35)$ & 0.428 & $0.84(0.58,1.21)$ & 0.350 \\
\hline $\begin{array}{l}\text { Polyunsaturated } \\
\text { fat }\end{array}$ & $0.97(0.78,1.21)$ & 0.778 & $1.07(0.86,1.32)$ & 0.564 & $1.15(0.93,1.43)$ & 0.203 & $0.99(0.61,1.62)$ & 0.974 \\
\hline Dairy fat & $0.89(0.72,1.10)$ & 0.283 & $1.13(0.79,1.61)$ & 0.501 & $1.17(0.62,2.22)$ & 0.631 & $0.75(0.44,1.27)$ & 0.287 \\
\hline Trans fat & $1.26(1.02,1.61)$ & 0.044 & $1.27(0.89,1.81)$ & 0.466 & $1.33(0.72,2.44)$ & 0.358 & \begin{tabular}{|l|}
$1.10(0.64,1.89)$ \\
\end{tabular} & 0.729 \\
\hline
\end{tabular}

$P$-value for significant test. $(P<0.050)$

Abbreviations: RR: relative risk, CI: confidence interval

\section{Dietary fat consumption and ovarian cancer subtypes}

Five studies involved dietary fat intake and risk of ovarian cancer subtypes. Significant positive association was found between total fat, saturated fat, trans-fat intake and serous ovarian tumor risk. High saturated fat intake was associated with a $34 \%$ increase in endometroid ovarian cancer risk. The RR for high animal fat intake was $1.36(95 \% \mathrm{CI}=1.08-1.73, P=0.011)$, suggesting a significant positive association between animal fat consumption and mucinous ovarian cancer risk. (Table 3)

\section{Subgroup and sensitivity analysis}

Subgroup analysis stratified by the geographic areas, study types and confounding factors of included studies was performed. Saturated fat $(\mathrm{RR}=1.20,95 \% \mathrm{CI}=1.04$ $1.39)$ and dairy fat $(\mathrm{RR}=1.37,95 \% \mathrm{CI}=1.05,1.79)$ intake could increase ovarian cancer risk in European populations; a positive trend was present among American populations, while an inverse trend was found in other populations (including Asians and South Americans). The conclusions of case-control and cohort studies were basically consistent; however, cohort studies were more inclined to a positive association between dietary fat intake and ovarian cancer risk, though no statistical significance was obtained. The summary results were modified by menopausal status, hormone replacement therapy, BMI, and pregnancy times. (Table 4-1 and Table 4-2.)

Sensitivity analysis showed that the results obtained for the association between saturated fat intake and ovarian cancer risk were significantly influenced by one study [29], which didn’t adjust hormone use and pregnancy times.

\section{Publication bias}

We found no evidence of publication bias with regard to dietary fat consumption and ovarian cancer risk by means of visual inspection of funnel plots and formal statistical tests, including Begg rank correlation test and Egger linear regression test (all $P>0.05$ ).

\section{DISCUSSION}

Dietary fat, as one of the most controversial dietary factors in nutritional epidemiology, might elevate the incidence of hormone related cancers, including breast, endometrial and ovarian cancers, but discrepant observational results have been reported. We thoroughly searched the literature, and found the incidence of two important cancer types, breast cancer and stomach cancer, were in relation to the high consumption of dietary fat. Breast cancer was traditionally considered to be linked with western lifestyles, other including prostate and colorectal cancers, and the inflammatory bowel diseases; IBD, Crohn's disease (CD). Stomach cancer was considered to be linked more with eastern lifestyle. The included two meta-analyses [7, 8] indicated positive associations between dietary fat intake and breast and stomach cancer. Considering the regional difference of these two cancers, we can conclude that the effect of dietary fat on cancer risk may be independent of the region. To define the effect of dietary fat on ovarian cancer risk, we conducted this meta-analysis to clear this research subject.

The results of this meta-analysis including case- 


\section{Dairy Fat and Ovarian Cancer Risk}

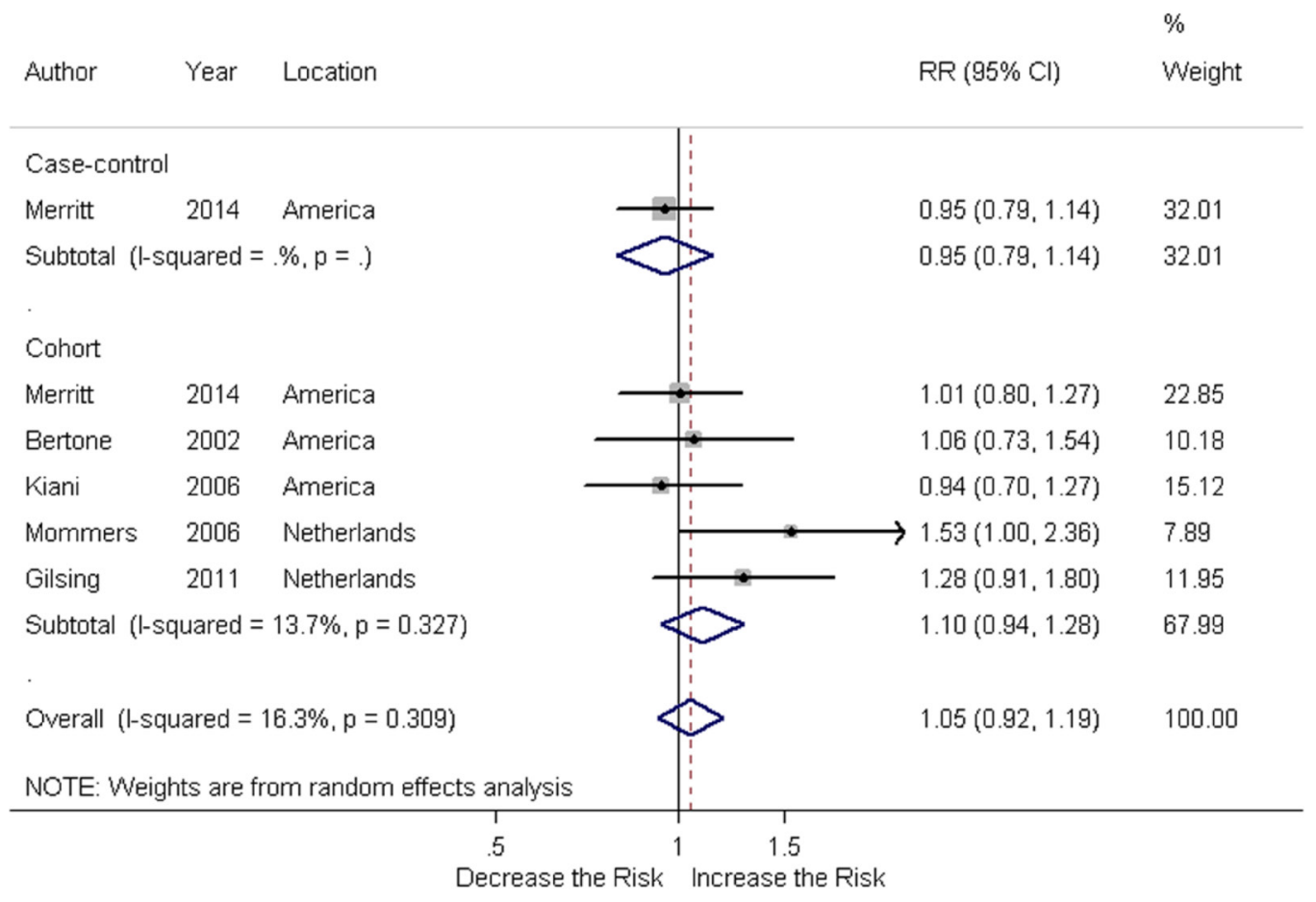

Figure 8: Relationship between dairy fat intake and ovarian cancer risk.

\section{Trans Fat and Ovarian Cancer Risk}

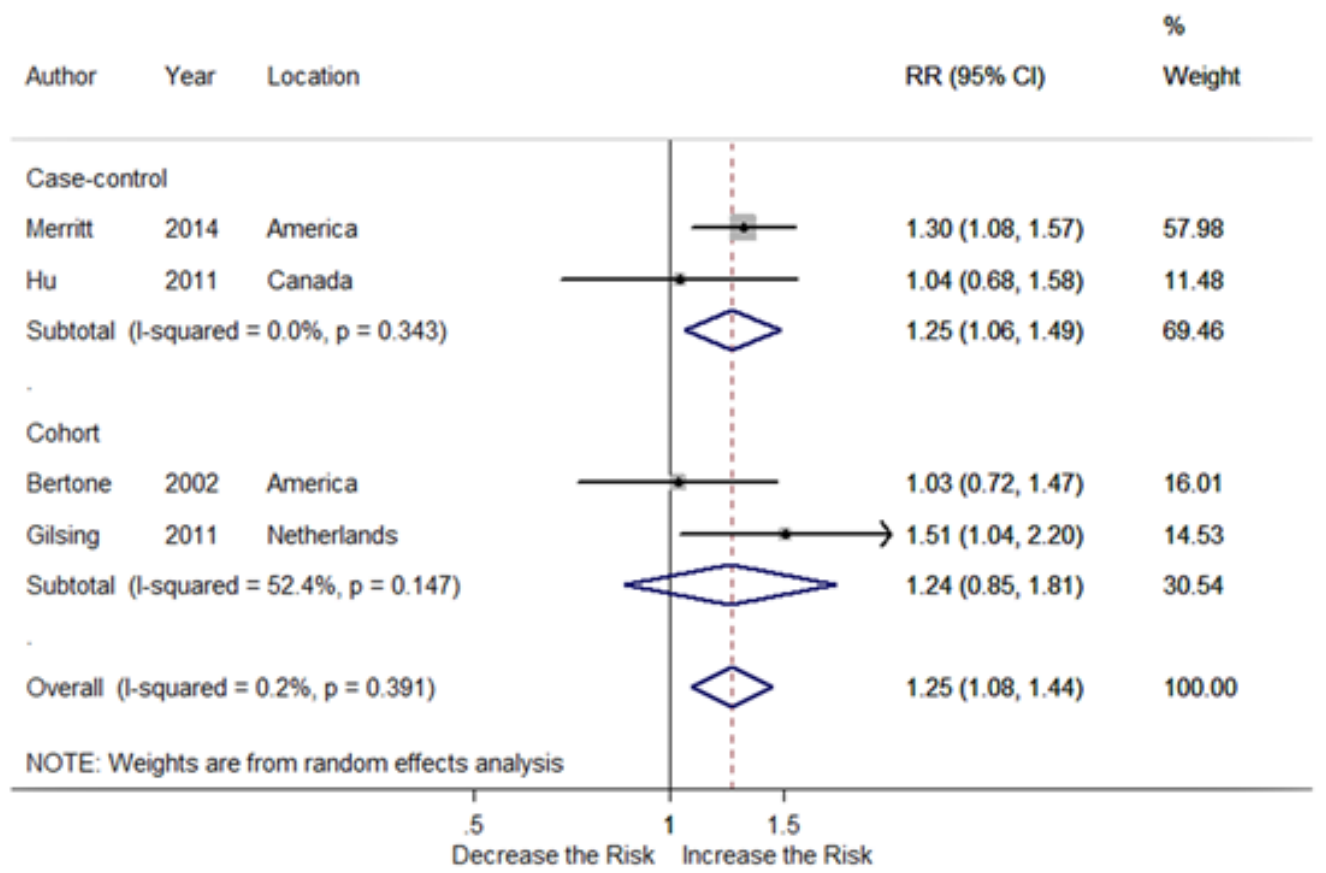

Figure 9: Relationship between trans fat intake and ovarian cancer risk. 
Table 4-1: Subgroup analysis based on study characteristics

\begin{tabular}{|c|c|c|c|c|c|c|}
\hline & \multicolumn{2}{|c|}{ Total fat } & \multicolumn{2}{|c|}{ Animal fat } & \multicolumn{2}{|c|}{ Plant fat } \\
\hline & $N$ & RR $(95 \% C I)$ & $N$ & RR $(95 \% C I)$ & $N$ & RR $(95 \%$ CI $)$ \\
\hline All studies & 17 & $\begin{array}{l}1.19(1.04,1.37) \\
\mathrm{I}^{2}=60.1 \%, \mathrm{p}=0.001\end{array}$ & 10 & $\begin{array}{l}1.21(0.99,1.47) \\
\mathrm{I}^{2}=68.6 \%, \mathrm{p}=0.001\end{array}$ & 9 & $\begin{array}{l}0.95(0.83,1.09) \\
\mathrm{I}^{2}=29.2 \%, \mathrm{p}=0.185\end{array}$ \\
\hline \multicolumn{7}{|l|}{ Study type } \\
\hline Case-control & 11 & \begin{tabular}{|l|}
$1.32(1.06,1.63)$ \\
$\mathrm{I}^{2}=69.8 \%, \mathrm{p}=0.000$
\end{tabular} & 5 & $\begin{array}{l}1.50(0.89,2.53) \\
\mathrm{I}^{2}=82.3 \%, \mathrm{p}=0.000\end{array}$ & 4 & $\begin{array}{l}0.96(0.81,1.12) \\
\mathrm{I}^{2}=0.0 \%, \mathrm{p}=0.858\end{array}$ \\
\hline Cohort & 6 & $\begin{array}{l}1.10(0.97,1.24) \\
\mathrm{I}^{2}=4.2 \%, \mathrm{p}=0.389 \\
\end{array}$ & 5 & $\begin{array}{l}1.09(0.93,1.28) \\
\mathrm{I}^{2}=28.4 \%, \mathrm{p}=0.232\end{array}$ & 5 & $\begin{array}{l}0.93(0.74,1.17) \\
\mathrm{I}^{2}=61.7 \%, \mathrm{p}=0.034\end{array}$ \\
\hline \multicolumn{7}{|c|}{ Geographic location or country } \\
\hline America & 7 & $\begin{array}{l}1.08(0.95,1.22) \\
\mathrm{I}^{2}=2.7 \%, \mathrm{p}=0.399 \\
\end{array}$ & 5 & $\begin{array}{l}1.13(0.96,1.34) \\
\mathrm{I}^{2}=27.9 \%, \mathrm{p}=0.235\end{array}$ & 4 & $\begin{array}{l}0,97(0.85,1.11) \\
\mathrm{I}^{2}=0.0 \%, \mathrm{p}=0.805\end{array}$ \\
\hline Europe & 4 & $\begin{array}{l}1.25(0.91 .1 .73) \\
\mathrm{I}^{2}=83.5 \%, \mathrm{P}=0.000 \\
\end{array}$ & 2 & $\begin{array}{l}1.08(0.81,1.44) \\
\mathrm{I}^{2}=58.2 \%, \mathrm{p}=0.012\end{array}$ & 2 & $\begin{array}{l}0.90(0.48,1.69) \\
\mathrm{I}^{2}=89.2 \%, \mathrm{p}=0.002\end{array}$ \\
\hline Others & 6 & $\begin{array}{l}1.33(0.98,1.82) \\
\mathrm{I}^{2}=63.1 \%, \mathrm{p}=0.019\end{array}$ & 3 & $\begin{array}{l}1.69(0.59,4.83) \\
\mathrm{I}^{2}=88.1 \%, \mathrm{p}=0.000\end{array}$ & 3 & $\begin{array}{l}0.88(0.62,1.09) \\
\mathrm{I}^{2}=0.0 \%, \mathrm{p}=0.796\end{array}$ \\
\hline \multicolumn{7}{|l|}{ Adjusted for } \\
\hline \multicolumn{7}{|l|}{ Total energy } \\
\hline Yes & 12 & \begin{tabular}{|l|}
$1.12(0.97,1.29)$ \\
$\mathrm{I}^{2}=48.8 \%, \mathrm{p}=0.034$
\end{tabular} & 5 & $\begin{array}{l}1.07(0.87,1.31) \\
\mathrm{I}^{2}=48.9 \%, \mathrm{p}=0.098\end{array}$ & 5 & $\begin{array}{l}0.90(0.70,1.16) \\
\mathrm{I}^{2}=63.2 \%, \mathrm{p}=0.028\end{array}$ \\
\hline No & 5 & \begin{tabular}{|l|}
$1.39(0.99,1.95)$ \\
$\mathrm{I}^{2}=62.3 \%, \mathrm{p}=0.001$
\end{tabular} & 5 & $\begin{array}{l}1.55(1.00,2.39) \\
\mathrm{I}^{2}=80.1 \%, \mathrm{p}=0.000\end{array}$ & 4 & $\begin{array}{l}0.97(0.83,1.13) \\
\mathrm{I}^{2}=0.0 \%, \mathrm{p}=0.936\end{array}$ \\
\hline \multicolumn{7}{|l|}{ Family history } \\
\hline Yes & 5 & \begin{tabular}{|l|}
$1.16(0.93,1.44)$ \\
$\mathrm{I}^{2}=44.7 \%, \mathrm{p}=0.143$
\end{tabular} & 4 & $\begin{array}{l}1.17(0.97,1.40) \\
\mathrm{I}^{2}=28.0 \%, \mathrm{p}=0.244\end{array}$ & 4 & $\begin{array}{l}0.96(0.84,1.10) \\
\mathrm{I}^{2}=29.2 \% \cdot \mathrm{p}=0.185\end{array}$ \\
\hline No & 12 & \begin{tabular}{|l|}
$1.20(1.00,1.43)$ \\
$\mathrm{I}^{2}=67.8 \%, \mathrm{p}=0.000$
\end{tabular} & 6 & $\begin{array}{l}1.27(0.89,1.82) \\
\mathrm{I}^{2}=79.3 \%, \mathrm{p}=0.000\end{array}$ & 5 & $\begin{array}{l}0.93(0.71,1.22) \\
\mathrm{I}^{2}=59.4 \%, \mathrm{p}=0.043\end{array}$ \\
\hline \multicolumn{7}{|l|}{ OC use } \\
\hline Yes & 12 & \begin{tabular}{|l|}
$1.28(1.09,1.51)$ \\
$\mathrm{I}^{2}=65.9 \%, \mathrm{p}=0.001$
\end{tabular} & 6 & $\begin{array}{l}1.11(0.97,1.29) \\
\mathrm{I}^{2}=37.1 \%, \mathrm{p}=0.159\end{array}$ & 6 & $\begin{array}{l}0.96(0.82,1.14) \\
\mathrm{I}^{2}=49.2 \%, \mathrm{p}=0.080 \\
\end{array}$ \\
\hline No & 5 & \begin{tabular}{|l|}
$0.98(0.79,1.22)$ \\
$\mathrm{I}^{2}=31.5 \%, \mathrm{p}=0.211$
\end{tabular} & 4 & $\begin{array}{l}1.49(0.69,3.21) \\
\mathrm{I}^{2}=84.2 \%, \mathrm{p}=0.000\end{array}$ & 3 & $\begin{array}{l}0.85(0.61,1.17) \\
\mathrm{I}^{2}=0.0 \%, \mathrm{p}=0.716\end{array}$ \\
\hline \multicolumn{7}{|l|}{ BMI } \\
\hline Yes & 6 & \begin{tabular}{|l|}
$1.57(1.24,1.99)$ \\
$\mathrm{I}^{2}=56.3 \%, \mathrm{p}=0.043$ \\
\end{tabular} & 3 & $\begin{array}{l}2.08(1.04,4.14) \\
\mathrm{I}^{2}=81.5 \%, \mathrm{p}=0.004\end{array}$ & 2 & $\begin{array}{l}1.00(0.81,1.25) \\
\mathrm{I}^{2}=29.2 \%, \mathrm{p}=0.185\end{array}$ \\
\hline No & 11 & \begin{tabular}{|l|}
$1.04(0.94,1.16)$ \\
$\mathrm{I}^{2}=17.3 \%, \mathrm{p}=0.284$ \\
\end{tabular} & 7 & $\begin{array}{l}1.03(0.90,1.18) \\
\mathrm{I}^{2}=22.0 \%, \mathrm{p}=0.261\end{array}$ & 7 & $\begin{array}{l}0.92(0.77,1.10) \\
\mathrm{I}^{2}=46.5 \%, \mathrm{p}=0.082 \\
\end{array}$ \\
\hline \multicolumn{7}{|c|}{ Menopausal status } \\
\hline Yes & 9 & \begin{tabular}{|l|}
$1.20(0.95,1.51)$ \\
$\mathrm{I}^{2}=75.2 \%, \mathrm{p}=0.000$
\end{tabular} & 4 & $\begin{array}{l}1.29(0.80,2.08) \\
\mathrm{I}^{2}=82.5 \%, \mathrm{p}=0.001\end{array}$ & 4 & $\begin{array}{l}1.08(0.90,1.30) \\
\mathrm{I}^{2}=8.4 \%, \mathrm{p}=0.351\end{array}$ \\
\hline No & 8 & \begin{tabular}{|l|}
$1.17(0.99,1.38)$ \\
$\mathrm{I}^{2}=38.7 \%, \mathrm{p}=0.121$
\end{tabular} & 6 & $\begin{array}{l}1.21(0.98,1.49) \\
\mathrm{I}^{2}=50.0 \%, \mathrm{p}=0.075\end{array}$ & 5 & $\begin{array}{l}0.89(0.76,1.05) \\
\mathrm{I}^{2}=25.7 \%, \mathrm{p}=0.185\end{array}$ \\
\hline \multicolumn{7}{|l|}{ Hormone use } \\
\hline Yes & 3 & \begin{tabular}{|l|}
$1.08(0.85,1.37)$ \\
$\mathrm{I}^{2}=41.7 \%, \mathrm{p}=0.180$
\end{tabular} & 2 & $\begin{array}{l}1.15(0.85,1.55) \\
\mathrm{I}^{2}=48.3 \%, 0.164 \\
\end{array}$ & 2 & $\begin{array}{l}0.99(0.81,1.21) \\
\mathrm{I}^{2}=0.0 \%, \mathrm{p}=0.982\end{array}$ \\
\hline No & 14 & \begin{tabular}{|l|}
$1.23(1.04,1.45)$ \\
$\mathrm{I}^{2}=66.6 \%, \mathrm{p}=0.000$
\end{tabular} & 8 & $\begin{array}{l}1.25(0.97,1.62) \\
\mathrm{I}^{2}=73.4 \%, \mathrm{p}=0.000\end{array}$ & 7 & $\begin{array}{l}0.92(0.76,1.11) \\
\mathrm{I}^{2}=46.7 \%, \mathrm{p}=0.081\end{array}$ \\
\hline \multicolumn{7}{|c|}{ Pregnancy times } \\
\hline Yes & 9 & \begin{tabular}{|l|}
$1.10(0.98,1.25)$ \\
$\mathrm{I}^{2}=15.7 \%, \mathrm{p}=0.303$
\end{tabular} & 6 & $\begin{array}{l}1.05(0.8,1.23) \\
\mathrm{I}^{2}=33.5 \%, \mathrm{p}=0.185\end{array}$ & 6 & $\begin{array}{l}0.90(0.73,1.11) \\
\mathrm{I}^{2}=0.0 \%, \mathrm{p}=0.990\end{array}$ \\
\hline No & 8 & \begin{tabular}{|l|}
$1.33(1.01,1.74)$ \\
$\mathrm{I}^{2}=78.2 \%, \mathrm{p}=0.000$
\end{tabular} & 4 & $\begin{array}{l}1.64(1.00,2.69) \\
\mathrm{I}^{2}=80.6 \%, \mathrm{p}=0.001\end{array}$ & 3 & $\begin{array}{l}1.00(0.83,1.21) \\
\mathrm{I}^{2}=55.4 \%, \mathrm{p}=0.047\end{array}$ \\
\hline
\end{tabular}


Table 4-2: Subgroup analysis based on study characteristics

\begin{tabular}{|c|c|c|c|c|c|c|}
\hline & \multicolumn{2}{|c|}{ Monounsaturated fat } & \multicolumn{2}{|c|}{ Polyunsaturated fat } & \multicolumn{2}{|c|}{ Dairy fat } \\
\hline & $N$ & RR (95\%CI) & $N$ & RR (95\%CI) & $N$ & RR $(95 \%$ CI $)$ \\
\hline All studies & 13 & $\begin{array}{l}0.98(0.87,1.09) \\
\mathrm{I}^{2}=42.6 \%, \mathrm{p}=0.052\end{array}$ & 13 & $\begin{array}{l}0.97(0.86,1.11) \\
\mathrm{I}^{2}=48.9 \%, \mathrm{p}=0.024\end{array}$ & 6 & $\begin{array}{l}1.05(0.92,1.19) \\
\mathrm{I}^{2}=16.3 \%, \mathrm{p}=0.309\end{array}$ \\
\hline \multicolumn{7}{|l|}{ Study type } \\
\hline Case-control & 8 & \begin{tabular}{|l|}
$0.96(0.83,1.12)$ \\
$\mathrm{I}^{2}=55.2 \%, \mathrm{p}=0.029$
\end{tabular} & 8 & $\begin{array}{l}0.93(0.82,1.05) \\
\mathrm{I}^{2}=26.7 \%, \mathrm{p}=0.216\end{array}$ & 1 & $0.95(0.79,1.14)$ \\
\hline Cohort & 5 & $\begin{array}{l}1.04(0.88,1.22) \\
\mathrm{I}^{2}=4.5, \mathrm{p}=0.381\end{array}$ & 5 & $\begin{array}{l}1.06(0.86,1.31) \\
\mathrm{I}^{2}=49.8 \%, \mathrm{p}=0.093\end{array}$ & 5 & $\begin{array}{l}1.10(0.94,1.28) \\
\mathrm{I}^{2}=13.7 \%, \mathrm{p}=0.327\end{array}$ \\
\hline \multicolumn{7}{|c|}{ Geographic location/country } \\
\hline America & 6 & $\begin{array}{l}0.98(0.85,1.13) \\
\mathrm{I}^{2}=0.0 \%, \mathrm{p}=0.507\end{array}$ & 6 & \begin{tabular}{|l|}
$0.97(0.76,1.25)$ \\
$\mathrm{I}^{2}=57.9 \%, \mathrm{p}=0.050$
\end{tabular} & 4 & $\begin{array}{l}0.98(0.86,1.10) \\
\mathrm{I}^{2}=0.0 \%, \mathrm{p} 0.937\end{array}$ \\
\hline Europe & 4 & $\begin{array}{l}0.92(0.77,1.10) \\
\mathrm{I}^{2}=56.4 \%, \mathrm{p}=0.076\end{array}$ & 4 & $\begin{array}{l}1.03(0.89,1.19) \\
\mathrm{I}^{2}=35.3 \%, \mathrm{p}=0.200\end{array}$ & 2 & $\begin{array}{l}1.37(1.05,1.79) \\
\mathrm{I}^{2}=0.0 \%, \mathrm{p}=0.524\end{array}$ \\
\hline Others & 3 & $\begin{array}{l}1.00(0.72,1.38) \\
\mathrm{I}^{2}=67.1 \%, \mathrm{p}=0.048\end{array}$ & 3 & $\begin{array}{l}0.92(0.65,1.32) \\
\mathrm{I}^{2}=68.8 \%, \mathrm{p}=0.041\end{array}$ & 0 & \\
\hline \multicolumn{7}{|l|}{ Adjust for } \\
\hline \multicolumn{7}{|l|}{ Total energy } \\
\hline Yes & 9 & \begin{tabular}{|l|}
$0.98(0.84,1.14)$ \\
$\mathrm{I}^{2}=47.8 \%, \mathrm{p}=0.063$
\end{tabular} & 9 & $\begin{array}{l}099(0.84,1.17) \\
\mathrm{I}^{2}=59.2 \%, \mathrm{p}=0.016\end{array}$ & 4 & $\begin{array}{l}1.03(0.87,1.23) \\
\mathrm{I}^{2}=30.4 \%, \mathrm{p}=0.230\end{array}$ \\
\hline No & 4 & \begin{tabular}{|l|}
$0.93(0.79,1.10)$ \\
$\mathrm{I}^{2}=32.4 \%, \mathrm{p}=0.218$
\end{tabular} & 4 & $\begin{array}{l}0.93(0.80,1.08) \\
\mathrm{I}^{2}=14.6 \%, \mathrm{p}=0.319\end{array}$ & 2 & $\begin{array}{l}1.10(0.88,1.37) \\
\mathrm{I}^{2}=21.3 \%, \mathrm{p}=0.260\end{array}$ \\
\hline \multicolumn{7}{|c|}{ Family history } \\
\hline Yes & 4 & \begin{tabular}{|l|}
$1.00(0.84,1.19)$ \\
$\mathrm{I}^{2}=29.0 \%, \mathrm{p}=0.238$ \\
\end{tabular} & 4 & $\begin{array}{l}0.92(0.73,1.15) \\
\mathrm{I}^{2}=60.8 \%, \mathrm{p}=0.054\end{array}$ & 2 & $\begin{array}{l}0.97(0.84,1.12) \\
\mathrm{I}^{2}=0.0 \%, \mathrm{p}=0.684\end{array}$ \\
\hline No & 9 & \begin{tabular}{l|}
$0.95(0.82,1.11)$ \\
$\mathrm{I}^{2}=50.6 \%, \mathrm{p}=0.048$
\end{tabular} & 9 & $\begin{array}{l}1.02(0.88,1.18) \\
\mathrm{I}^{2}=42.9 \%, \mathrm{p}=0.092\end{array}$ & 4 & $\begin{array}{l}1.15(0.94,1.41) \\
\mathrm{I}^{2}=25.1 \%, \mathrm{p}=0.684\end{array}$ \\
\hline \multicolumn{7}{|l|}{ OC use } \\
\hline Yes & 8 & \begin{tabular}{|l|}
$0.99(0.89,1.10)$ \\
$\mathrm{I}^{2}=26.4 \%, \mathrm{p}=0.227$
\end{tabular} & 8 & $\begin{array}{l}1.00(0.86,1.15) \\
\mathrm{I}^{2}=55.9 \%, \mathrm{p}=0.034\end{array}$ & 5 & $\begin{array}{l}1.08(0.93,1.25) \\
\mathrm{I}^{2}=27.3 \%, \mathrm{p}=0.239\end{array}$ \\
\hline No & 5 & \begin{tabular}{|l|}
$0.90(0.68,1.20)$ \\
$\mathrm{I}^{2}=61.0 \%, \mathrm{p}=0.036$
\end{tabular} & 5 & $\begin{array}{l}0.93(0.71,1.21) \\
\mathrm{I}^{2}=53.8 \%, \mathrm{p}=0.070\end{array}$ & 1 & $0.94(0.70,1.27)$ \\
\hline \multicolumn{7}{|l|}{ BMI } \\
\hline Yes & 9 & $\begin{array}{l}1.20(0.94,1.52) \\
\mathrm{I}^{2}=0.0 \%, \mathrm{p}=0.709\end{array}$ & 9 & $\begin{array}{l}1.27(1.03,1.58) \\
\mathrm{I}^{2}=0.0 \%, \mathrm{p}=0.984\end{array}$ & 1 & $0.94(0.70,1.27)$ \\
\hline No & 4 & \begin{tabular}{|l|}
$0.93(0.83,1.05)$ \\
$\mathrm{I}^{2}=47.0 \%, \mathrm{p}=0.058$
\end{tabular} & 4 & $\begin{array}{l}0.93(0.82,1.05) \\
\mathrm{I}^{2}=49.8 \%, \mathrm{p}=0.043\end{array}$ & 5 & $\begin{array}{l}1.08(0.93,1.25) \\
\mathrm{I}^{2}=27.3 \%, \mathrm{p}=0.239\end{array}$ \\
\hline \multicolumn{7}{|c|}{ Menopausal status } \\
\hline Yes & 6 & $\begin{array}{l}1.01(0.83,1.23) \\
\mathrm{I}^{2}=53.9 \% \mathrm{p}=0.070 \\
\end{array}$ & 5 & $\begin{array}{l}1.10(0.87,1.39) \\
\mathrm{I}^{2}=53.3 \%, \mathrm{p}=0.093\end{array}$ & 3 & $\begin{array}{l}1.00(0.85,1.18) \\
\mathrm{I}^{2}=0.0 \%, \mathrm{p}=0.876\end{array}$ \\
\hline No & 7 & \begin{tabular}{|l|}
$0.93(0.81,1.08)$ \\
$\mathrm{I}^{2}=38.2 \%, \mathrm{p}=0.138$
\end{tabular} & 8 & $\begin{array}{l}0.92(0.82,1.02) \\
\mathrm{I}^{2}=16.6 \%, \mathrm{p}=0.299\end{array}$ & 3 & $\begin{array}{l}1.17(0.88,1.57) \\
\mathrm{I}^{2}=62.6 \%, \mathrm{p}=0.069\end{array}$ \\
\hline \multicolumn{7}{|l|}{ Hormone use } \\
\hline Yes & 2 & $\begin{array}{l}1.05(0.79,1.39) \\
\mathrm{I}^{2}=0.0 \%, \mathrm{p}=0.847\end{array}$ & 2 & $\begin{array}{l}1.22(0.95,1.55) \\
\mathrm{I}^{2}=0.0 \%, \mathrm{p}=0.642 \\
\end{array}$ & 2 & $\begin{array}{l}0.98(0.78,1.24) \\
\mathrm{I}^{2}=0.0 \%, \mathrm{p}=0.622\end{array}$ \\
\hline No & 11 & $\begin{array}{l}0.96(0.84,1.09) \\
\mathrm{I}^{2}=52.0 \%, \mathrm{p}=0.027\end{array}$ & 11 & \begin{tabular}{|l|}
$0.95(0.83,1.08)$ \\
$\mathrm{I}^{2}=52.3 \%, \mathrm{p}=0.026$
\end{tabular} & 4 & $\begin{array}{l}1.10(0.91,1.32) \\
\mathrm{I}^{2}=45.5 \%, \mathrm{p}=0.139\end{array}$ \\
\hline \multicolumn{7}{|c|}{ Pregnancy times } \\
\hline Yes & 8 & $\begin{array}{l}1.02(0.89,1.17) \\
\mathrm{I}^{2}=38.6 \%, \mathrm{p}=0.122\end{array}$ & 8 & $\begin{array}{l}0.93(0.78,1.12) \\
\mathrm{I}^{2}=62.9 \%, \mathrm{p}=0.009\end{array}$ & 3 & $\begin{array}{l}1.02(0.88,1.18) \\
\mathrm{I}^{2}=12.2 \%, \mathrm{p}=0.320\end{array}$ \\
\hline No & 5 & $\begin{array}{l}0.87(0.77,0.98) \\
\mathrm{I}^{2}=0.0 \%, \mathrm{p}=0.047\end{array}$ & 5 & $\begin{array}{l}1.02(0.90,1.17) \\
\mathrm{I}^{2}=0.0 \%, \mathrm{p}=0.405\end{array}$ & 3 & $\begin{array}{l}1.11(0.85,1.46) \\
\mathrm{I}^{2}=40.6 \%, \mathrm{p}=0.186\end{array}$ \\
\hline
\end{tabular}

$P$-value for heterogeneity within each subgroup. 
control and cohort studies indicated that consumption of total dietary fat and trans fat increased the risk of ovarian cancer. These findings were consistent with the results in specific epidemical studies in NIH-AARP cohort study [35] and an earlier meta-analysis [11], which reported positive associations between total fat intake and EOC risk. Contrasted with our meta-analysis, a pooled analysis [12], three cohort studies [34, 39, 40] and several casecontrol studies [20, 24, 27, 29] observed no association between total fat consumption and EOC risk. Consistent with a pooled analysis [12] of 12 cohort studies, we observed no significant associations of consumption of dietary fat and risk of ovarian cancer subtypes. There was also no relevance in the associations with dietary fat intake and the risk of different histological subtypes of EOC in an American case-control study [27] and an Italian case-control study [30]. Limited studies involved in associations between dietary fat intakes and EOC risk attribute to different histological subtypes of EOC. Merritt [34] (EPIC) reported high intake of polyunsaturated fatty acids can increase risk for serous EOC. Blank [35] observed an increased risk of serous EOC by total energy from animal fat and inverse associations with risk of EOC observed for the intakes of plant fat and polyunsaturated FAs. Beral [49] reported that serous subtype appeared to have more consistent global distribution, followed by endometrioid subtype, whereas mucinous and clear cell subtypes varied significantly across countries. Among these four main subtypes, the clear cell subtype was least studied, which has higher prevalence in Asia [50-52] and was more frequently found in younger women [52]. In the current meta-analysis, there was only one study [27] from America involving ovarian clear cell carcinoma (OCCC). In addition, the survival outcome of OCCC was comparable to that of serous EOC in terms of early-stage disease [50, 53-55], but worse with respect to advancedstage disease [56-62]. Therefore, more studies, including epidemiological and clinical studies, should be carried out in Asia and other "new regions" (Central and South America, Africa) [63].

Subgroup analysis based on the characteristics of included studies suggested that menopausal status, hormone replacement therapy, BMI, and pregnancy times can modify the association between dietary fat intake and ovarian cancer risk. These factors are related to exposure to estrogens [64, 65]. A reanalysis [49] of epidemiological data suggested estrogen monotherapy or estrogen and progesterone combination therapy could elevate the risk of ovarian cancer, specifically serous or endometroid tumors. In ovarian tissues, estrogen receptors are also expressed [66]; the ratios of estrogen-DNA adduct depurination to estrogen metabolites and conjugates in ovarian cancer cases are significantly higher than controls [67]. We speculated that hormonal pathways might play a positive role in the development of ovarian cancer. High consumption of dietary fat could stimulate the secretion of extra ovarian estrogen $[68,69]$, which can exert tumorpromoting activity via mitogenic effects on ER $\alpha$ - positive $[70,71]$ or negative [72] tumor cells, therefore increasing the risk of ovarian cancer [66]. What's more, obese women may suffer from insulin resistance, and concurrent hyperinsulinemia with excess insulin-like growth factor-1 receptor (IGF-1) could additionally induce androgen steroidogenesis [73] and lead to tumor development [74, 75].

An important highlight of our meta-analysis is that we analyzed the association between dietary fat intake and the risk of ovarian cancer subtypes. We found that serous ovarian cancer incidence was more susceptible to dietary fat intake. However, these results should be interpreted with caution. The insufficient number of included cases and potential misclassification of pathological subtypes may contribute to the statistical difference observed.

Several limitations of this meta-analysis should be considered. First, there was substantial heterogeneity across studies assessing the associations of dietary fat intake with ovarian cancer risk. Considering the varieties of the characteristics of included populations, and study designs and types, the existence of substantial heterogeneity was reasonable, and we conducted subgroup analysis to reduce its effect on the results. Second, misclassification bias, which stemmed mainly from the misclassification of dietary assessments and pathological subtypes of ovarian cancer, should be paid enough attention to. Misclassification of dietary assessments may result from the differences across nutrient databases or designed questionnaires. Diagnosis, pathology review, and classification methods could cause misclassification bias of pathological subtypes of ovarian cancer. Third, we couldn't rule out the effects of confounding factors and various statistical biases on our results. Furthermore, controls and confounding factor adjustment methods across individual studies were not consistent. With more and more basic and clinical researches in recent years, and the increasing understanding of the relationship between diet and health, the confounding factors controlled have markedly increased in number, and bias was inevitable. Forth, although no publication bias was found, its possible effect cannot be totally excluded.

In conclusion, the present meta-analysis of casecontrol and cohort studies indicate that increased consumption of total fat, saturated fat and trans-fat may be associated with an increased risk of ovarian cancer. Among the dietary fats, saturated fats can significantly increase serous and endometroid ovarian cancer, with the risk of serous ovarian cancer more susceptible to dietary fat intake. In addition, subgroup analysis data suggested that menopausal status, hormone replacement therapy, BMI, and pregnancy times may serve as potential effect modifiers. Future studies should focus more on specific pathological subtypes of ovarian cancer as well as the influence of molecular mechanisms and genetic factors on 
the association of dietary fat and ovarian cancer.

\section{MATERIALS AND METHODS}

\section{Search strategy}

We obtained the literature published in any language to December 2015 by fully searching the PubMed database. The search terms used were "diet", "dietary fat" in combination with "ovarian cancer," "ovarian neoplasm" or "ovarian carcinoma", without restrictions. In addition, we reviewed the reference lists of retrieved studies and recent reviews to supplement electronic database searches.

\section{Study selection}

Study selection included initial screening of titles or abstracts, and a second one for full texts. Studies were eligible for inclusion if they met the following criteria: 1) observational studies which enrolled patients with proven epithelial ovarian cancers excluding tumors of "borderline malignant potential " histopathology, 2) patients enrolled were adults ( $\geq 18$ yr of age), 3 ) studies containing available data showing association(s) between intake of dietary fat (total, saturated, animal, dairy or unsaturated fat) and ovarian cancer, and 4) odds ratio or relative risk (RR) with 95\% confidence interval (CI) for each variable or availability of raw data to calculate these parameters.

\section{Data extraction}

All data were extracted with a data-collection form. Information was recorded as follows: last name of the first author, publication year, study population, period, country, sample size; risk estimate from multivariable model for the highest versus lowest category of dietary fat intake with the corresponding 95\% CI; statistical adjustment for the main confounding factors of interest.

Data extraction and study selection were performed by 3 authors (Qiu WL, Lu H and Qi YN) independently. Any disagreements were resolved by discussion.

\section{Statistical methods}

The association between dietary fat consumption and the risk of ovarian cancer was our main analytical object. Dietary fats in this meta-analysis were defined as total fat, animal fat, plant fat, dairy fat, saturated fat, monounsaturated fat, polyunsaturated fat and trans-fat. Relative risk (RR) was used as the common measure of association in this meta-analysis, and the random-effects model was selected to calculate summary RRs and 95\% CIs associated with dietary fats. Q statistic (significance level at $P<0.10$ ) and $\mathrm{I}^{2}$ statistic, a quantitative measure of inconsistency across studies [13], were applied for heterogeneity assessment of RRs across studies. Subgroup analyses stratified by geographic region (country), study type, and study characteristics were carried out to investigate potential sources of heterogeneity. Sensitivity analyses were performed by excluding one study at a time to assess the influence of a single study on the overall risk estimate. Publication bias was assessed with funnel plots, Egger's test [14], and Begg's test [15] (all $P>0.05$ ). The Stata version 12.0 software (StataCorp) was used for statistical analyses.

\section{Abbreviations}

EOC: epithelial ovarian cancer; RR, relative risk; OR, odds ratio; CI, confidential interval; BMI: body mass index.

\section{ACKNOWLEDGEMENTS}

Xiuwen Wang and Wenlong Qiu participated in the design of this article. Wenlong Qiu, Heng Lu and Yana Qi participated in abstracting the data and Wenlong Qiu performed statistical analysis. Xiuwen Wang and Wenlong Qiu wrote the paper. All authors read and approved the final article.

\section{CONFLICTS OF INTEREST}

There is no conflict of interest of this study.

\section{FUNDING}

This work was financially supported by the Science and Technology Research Project Foundation of Shandong Province (No. 2012GSF11845), the National Nature Science Foundation of China (No. 81372530), and the Science and Technology Plan Project Foundation of Jinan City (No. 201303047).

\section{REFERENCES}

1. Permuth-Wey J, and Sellers TA. Epidemiology of ovarian cancer. Methods Mol Biol. 2009; 472: 413-37.

2. Jemal A, Bray F, Center MM, Ferlay J, Ward E, and Forman D. Global cancer statistics. CA Cancer J Clin. 2011; 61: 6990.

3. Allen TW. Guide to clinical preventive services. Report of the US Preventive Services Task Force. J Am Osteopath Assoc. 1991; 91: 281-9.

4. Siegel R, Naishadham D, and Jemal A. Cancer statistics, 2012. CA Cancer J Clin. 2012; 62: 10-29.

5. Armstrong B, and Doll R. Environmental factors and cancer 
incidence and mortality in different countries, with special reference to dietary practices. Int J Cancer. 1975; 15: 61731.

6. Parkin DM, Bray F, Ferlay J, and Pisani P. Global cancer statistics, 2002. CA Cancer J Clin. 2005; 55: 74-108.

7. Cao Y, Hou L, and Wang W. Dietary total fat and fatty acids intake, serum fatty acids and risk of breast cancer: A meta-analysis of prospective cohort studies. Int J Cancer. 2016; 138: 1894-904.

8. Han J, Jiang Y, Liu X, Meng Q, Xi Q, Zhuang Q, Han Y, Gao Y, Ding Q, and Wu G. Dietary Fat Intake and Risk of Gastric Cancer: A Meta-Analysis of Observational Studies. PLoS One. 2015; 10: e0138580.

9. Prentice RL, and Sheppard L. Dietary fat and cancer: consistency of the epidemiologic data, and disease prevention that may follow from a practical reduction in fat consumption. Cancer Causes Control. 1990; 1: 81-97; discussion 99-109.

10. Hou R, Wu QJ, Gong TT, and Jiang L. Dietary fat and fatty acid intake and epithelial ovarian cancer risk: evidence from epidemiological studies. Oncotarget. 2015; 6:43099-119. doi: 10.18632/oncotarget.5525.

11. Huncharek M, and Kupelnick B. Dietary fat intake and risk of epithelial ovarian cancer: a meta-analysis of 6,689 subjects from 8 observational studies. Nutr Cancer. 2001; 40: 87-91.

12. Genkinger JM, Hunter DJ, Spiegelman D, Anderson KE, Beeson WL, Buring JE, Colditz GA, Fraser GE, Freudenheim JL, Goldbohm RA, Hankinson SE, Koenig $\mathrm{KL}$, Larsson SC, et al. A pooled analysis of 12 cohort studies of dietary fat, cholesterol and egg intake and ovarian cancer. Cancer Causes Control. 2006; 17: 273-85.

13. Higgins JP, Thompson SG, Deeks JJ, and Altman DG. Measuring inconsistency in meta-analyses. BMJ. 2003; 327: 557-60.

14. Egger M, Davey SG, Schneider M, and Minder C. Bias in meta-analysis detected by a simple, graphical test. BMJ. 1997; 315: 629-34.

15. Begg CB, and Mazumdar M. Operating characteristics of a rank correlation test for publication bias. Biometrics. 1994; 50: 1088-101.

16. Cramer DW, Welch WR, Hutchison GB, Willett W, and Scully RE. Dietary animal fat in relation to ovarian cancer risk. Obstet Gynecol. 1984; 63: 833-8.

17. Risch HA, Jain M, Marrett LD, and Howe GR. Dietary fat intake and risk of epithelial ovarian cancer. J Natl Cancer Inst. 1994; 86: 1409-15.

18. Risch HA, Marrett LD, Jain M, and Howe GR. Differences in risk factors for epithelial ovarian cancer by histologic type. Results of a case-control study. Am J Epidemiol. 1996; 144: 363-72.

19. Shu XO, Gao YT, Yuan JM, Ziegler RG, and Brinton LA. Dietary factors and epithelial ovarian cancer. Br J Cancer.
1989; 59: 92-6.

20. Slattery ML, Schuman KL, West DW, French TK, and Robison LM. Nutrient intake and ovarian cancer. Am J Epidemiol. 1989; 130: 497-502.

21. Tzonou A, Hsieh CC, Polychronopoulou A, Kaprinis G, Toupadaki N, Trichopoulou A, Karakatsani A, and Trichopoulos D. Diet and ovarian cancer: a case-control study in Greece. Int J Cancer. 1993; 55: 411-4.

22. La Vecchia C, Decarli A, Negri E, Parazzini F, Gentile A, Cecchetti G, Fasoli M, and Franceschi S. Dietary factors and the risk of epithelial ovarian cancer. J Natl Cancer Inst. 1987; 79: 663-9.

23. Webb PM, Bain CJ, Purdie DM, Harvey PW, and Green A. Milk consumption, galactose metabolism and ovarian cancer (Australia). Cancer Causes Control. 1998; 9: 637-44.

24. Pan SY, Ugnat AM, Mao Y, Wen SW, and Johnson KC. A case-control study of diet and the risk of ovarian cancer. Cancer Epidemiol Biomarkers Prev. 2004; 13: 1521-7.

25. Zhang M, Yang ZY, Binns CW, and Lee AH. Diet and ovarian cancer risk: a case-control study in China. Br J Cancer. 2002; 86: 712-7.

26. McCann SE, Freudenheim JL, Marshall JR, and Graham S. Risk of human ovarian cancer is related to dietary intake of selected nutrients, phytochemicals and food groups. J Nutr. 2003; 133: 1937-42.

27. Merritt MA, Cramer DW, Missmer SA, Vitonis AF, Titus LJ, and Terry KL. Dietary fat intake and risk of epithelial ovarian cancer by tumour histology. Br J Cancer. 2014; 110: 1392-401.

28. Zhang M, Lee AH, and Binns CW. Reproductive and dietary risk factors for epithelial ovarian cancer in China. Gynecol Oncol. 2004; 92: 320-6.

29. Salazar-Martinez E, Lazcano-Ponce EC, Gonzalez LG, Escudero-De 1RP, and Hernandez-Avila M. Nutritional determinants of epithelial ovarian cancer risk: a case-control study in Mexico. Oncology. 2002; 63: 151-7.

30. Chiaffarino F, Parazzini F, Bosetti C, Franceschi S, Talamini R, Canzonieri V, Montella M, Ramazzotti V, Franceschi S, and La Vecchia C. Risk factors for ovarian cancer histotypes. Eur J Cancer. 2007; 43: 1208-13.

31. Hu J, La Vecchia C, de Groh M, Negri E, Morrison H, and Mery L. Dietary transfatty acids and cancer risk. Eur J Cancer Prev. 2011; 20: 530-8.

32. Merritt MA, Poole EM, Hankinson SE, Willett WC, and Tworoger SS. Dairy food and nutrient intake in different life periods in relation to risk of ovarian cancer. Cancer Causes Control. 2014; 25: 795-808.

33. Bertone ER, Rosner BA, Hunter DJ, Stampfer MJ, Speizer FE, Colditz GA, Willett WC, and Hankinson SE. Dietary fat intake and ovarian cancer in a cohort of US women. Am J Epidemiol. 2002; 156: 22-31.

34. Merritt MA, Riboli E, Weiderpass E, Tsilidis KK, Overvad K, Tjonneland A, Hansen L, Dossus L, Fagherazzi G, 
Baglietto L, Fortner RT, Ose J, Steffen A, et al. Dietary fat intake and risk of epithelial ovarian cancer in the European Prospective Investigation into Cancer and Nutrition. Cancer Epidemiol. 2014; 38: 528-37.

35. Blank MM, Wentzensen N, Murphy MA, Hollenbeck A, and Park Y. Dietary fat intake and risk of ovarian cancer in the NIH-AARP Diet and Health Study. Br J Cancer. 2012; 106: 596-602.

36. Kiani F, Knutsen S, Singh P, Ursin G, and Fraser G. Dietary risk factors for ovarian cancer: the Adventist Health Study (United States). Cancer Causes Control. 2006; 17: 137-46.

37. Mommers M, Schouten LJ, Goldbohm RA, and van den Brandt PA. Dairy consumption and ovarian cancer risk in the Netherlands Cohort Study on Diet and Cancer. Br J Cancer. 2006; 94: 165-70.

38. Kushi LH, Mink PJ, Folsom AR, Anderson KE, Zheng W, Lazovich D, and Sellers TA. Prospective study of diet and ovarian cancer. Am J Epidemiol. 1999; 149: 21-31.

39. Chang ET, Lee VS, Canchola AJ, Clarke CA, Purdie DM, Reynolds P, Anton-Culver H, Bernstein L, Deapen D, Peel D, Pinder R, Ross RK, Stram DO, et al. Diet and risk of ovarian cancer in the California Teachers Study cohort. Am J Epidemiol. 2007; 165: 802-13.

40. Gilsing AM, Weijenberg MP, Goldbohm RA, van den Brandt PA, and Schouten LJ. Consumption of dietary fat and meat and risk of ovarian cancer in the Netherlands Cohort Study. Am J Clin Nutr. 2011; 93: 118-26.

41. Mori M, Harabuchi I, Miyake H, Casagrande JT, Henderson BE, and Ross RK. Reproductive, genetic, and dietary risk factors for ovarian cancer. Am J Epidemiol. 1988; 128: 7717.

42. Mori M, and Miyake H. Dietary and other risk factors of ovarian cancer among elderly women. Jpn J Cancer Res. 1988; 79: 997-1004.

43. Engle A, Muscat JE, and Harris RE. Nutritional risk factors and ovarian cancer. Nutr Cancer. 1991; 15: 239-47.

44. Tomao S, Taggi F, Sberna RC, and Villani C. Ovarian cancer and dietary habits. Eur J Gynaecol Oncol. 1992; 13: 91-5.

45. Bosetti C, Negri E, Franceschi S, Pelucchi C, Talamini R, Montella M, Conti E, and La Vecchia C. Diet and ovarian cancer risk: a case-control study in Italy. Int J Cancer. 2001; 93: 911-5.

46. Pirozzo S, Purdie D, Kuiper-Linley M, Webb P, Harvey P, Green A, and Bain C. Ovarian cancer, cholesterol, and eggs: a case-control analysis. Cancer Epidemiol Biomarkers Prev. 2002; 11: 1112-4.

47. Parazzini F, Chiaffarino F, Negri E, Surace M, Benzi G, Franceschi S, Fedele L, and La Vecchia C. Risk factors for different histological types of ovarian cancer. Int J Gynecol Cancer. 2004; 14: 431-6.

48. Lubin F, Chetrit A, Modan B, and Freedman LS. Dietary intake changes and their association with ovarian cancer risk. J Nutr. 2006; 136: 2362-7.
49. Beral V, Gaitskell K, Hermon C, Moser K, Reeves G, and Peto R. Menopausal hormone use and ovarian cancer risk: individual participant meta-analysis of 52 epidemiological studies. Lancet. 2015; 385: 1835-42.

50. Chan JK, Teoh D, Hu JM, Shin JY, Osann K, and Kapp DS. Do clear cell ovarian carcinomas have poorer prognosis compared to other epithelial cell types? A study of 1411 clear cell ovarian cancers. Gynecol Oncol. 2008; 109: 3706.

51. Orezzoli JP, Russell AH, Oliva E, Del CMG, Eichhorn J, and Fuller AF. Prognostic implication of endometriosis in clear cell carcinoma of the ovary. Gynecol Oncol. 2008; 110: 336-44.

52. Tay SK, and Cheong MA. Evidence for ethnic and environmental contributions to frequency of ovarian clear cell carcinoma. Aust N Z J Obstet Gynaecol. 2014; 54: 225 30.

53. Lee YY, Kim TJ, Kim MJ, Kim HJ, Song T, Kim MK, Choi CH, Lee JW, Bae DS, and Kim BG. Prognosis of ovarian clear cell carcinoma compared to other histological subtypes: a meta-analysis. Gynecol Oncol. 2011; 122: 5417.

54. Higashi M, Kajiyama H, Shibata K, Mizuno M, Mizuno K, Hosono S, Kawai M, Nakanishi T, Nagasaka T, and Kikkawa F. Survival impact of capsule rupture in stage I clear cell carcinoma of the ovary in comparison with other histological types. Gynecol Oncol. 2011; 123: 474-8.

55. Suh DH, Park JY, Lee JY, Kim BG, Lim MC, Kim JW, Bae DS, Park SY, Nam JH, Kim K, No JH, and Kim YB. The clinical value of surgeons' efforts of preventing intraoperative tumor rupture in stage I clear cell carcinoma of the ovary: A Korean multicenter study. Gynecol Oncol. 2015; 137: 412-7.

56. Takano M, Kikuchi Y, Yaegashi N, Kuzuya K, Ueki M, Tsuda H, Suzuki M, Kigawa J, Takeuchi S, Tsuda H, Moriya T, and Sugiyama T. Clear cell carcinoma of the ovary: a retrospective multicentre experience of 254 patients with complete surgical staging. Br J Cancer. 2006; 94: 1369-74.

57. Ye S, Yang J, You Y, Cao D, Bai H, Lang J, Chen J, and Shen K. Comparative study of ovarian clear cell carcinoma with and without endometriosis in People's Republic of China. Fertil Steril. 2014; 102: 1656-62.

58. Shevchuk MM, Winkler-Monsanto B, Fenoglio CM, and Richart RM. Clear cell carcinoma of the ovary: a clinicopathologic study with review of the literature. Cancer. 1981; 47: 1344-51.

59. Jenison EL, Montag AG, Griffiths CT, Welch WR, Lavin PT, Greer J, and Knapp RC. Clear cell adenocarcinoma of the ovary: a clinical analysis and comparison with serous carcinoma. Gynecol Oncol. 1989; 32: 65-71.

60. Goff BA, de la Cuesta R S, Muntz HG, Fleischhacker D, Ek M, Rice LW, Nikrui N, Tamimi HK, Cain JM, Greer $\mathrm{BE}$, and Fuller AF Jr. Clear cell carcinoma of the ovary: a 
distinct histologic type with poor prognosis and resistance to platinum-based chemotherapy in stage III disease. Gynecol Oncol. 1996; 60: 412-7.

61. Sugiyama T, Kamura T, Kigawa J, Terakawa N, Kikuchi Y, Kita T, Suzuki M, Sato I, and Taguchi K. Clinical characteristics of clear cell carcinoma of the ovary: a distinct histologic type with poor prognosis and resistance to platinum-based chemotherapy. Cancer. 2000; 88: 2584-9.

62. Makar AP, Baekelandt M, Trope CG, and Kristensen GB. The prognostic significance of residual disease, FIGO substage, tumor histology, and grade in patients with FIGO stage III ovarian cancer. Gynecol Oncol. 1995; 56: 175-80.

63. Shih WJ. Clinical trials for drug registrations in AsianPacific countries: proposal for a new paradigm from a statistical perspective. Control Clin Trials. 2001; 22: 35766.

64. Hunn J, and Rodriguez GC. Ovarian cancer: etiology, risk factors, and epidemiology. Clin Obstet Gynecol. 2012; 55: 3-23.

65. Salehi F, Dunfield L, Phillips KP, Krewski D, and Vanderhyden BC. Risk factors for ovarian cancer: an overview with emphasis on hormonal factors. J Toxicol Environ Health B Crit Rev. 2008; 11: 301-21.

66. Lukanova A, and Kaaks R. Endogenous hormones and ovarian cancer: epidemiology and current hypotheses. Cancer Epidemiol Biomarkers Prev. 2005; 14: 98-107.

67. Zahid M, Beseler CL, Hall JB, LeVan T, Cavalieri EL, and Rogan EG. Unbalanced estrogen metabolism in ovarian cancer. Int J Cancer. 2014; 134: 2414-23.
68. Hill MJ, Goddard P, and Williams RE. Gut bacteria and aetiology of cancer of the breast. Lancet. 1971; 2: 472-3.

69. Wu AH, Pike MC, and Stram DO. Meta-analysis: dietary fat intake, serum estrogen levels, and the risk of breast cancer. J Natl Cancer Inst. 1999; 91: 529-34.

70. Gallo D, De Stefano I, Grazia PM, Scambia G, and Ferrandina G. Estrogen receptor beta in cancer: an attractive target for therapy. Curr Pharm Des. 2012; 18: 2734-57.

71. Ribeiro JR, and Freiman RN. Estrogen signaling crosstalk: Implications for endocrine resistance in ovarian cancer. $\mathrm{J}$ Steroid Biochem Mol Biol. 2014; 143: 160-73.

72. Ciucci A, Zannoni GF, Buttarelli M, Lisi L, Travaglia D, Martinelli E, Scambia G, and Gallo D. Multiple direct and indirect mechanisms drive estrogen-induced tumor growth in high grade serous ovarian cancers. Oncotarget. 2016; 7:8155-71. doi: 10.18632/oncotarget.6943.

73. Kaaks R, and Lukanova A. Energy balance and cancer: the role of insulin and insulin-like growth factor-I. Proc Nutr Soc. 2001; 60: 91-106.

74. Khandwala HM, McCutcheon IE, Flyvbjerg A, and Friend KE. The effects of insulin-like growth factors on tumorigenesis and neoplastic growth. Endocr Rev. 2000; 21: 215-44.

75. Becker S, Dossus L, and Kaaks R. Obesity related hyperinsulinaemia and hyperglycaemia and cancer development. Arch Physiol Biochem. 2009; 115: 86-96. 